\title{
Applying new fixed point theorems on fractional and ordinary differential equations
}

\section{Erdal Karapınar ${ }^{1 *}$ (D), Thabet Abdeljawad ${ }^{2}$ and Fahd Jarad ${ }^{3}$}

\section{"Correspondence:}

erdalkarapinar@yahoo.com

'Department of Medical Research,

China Medical University, Taichung,

Taiwan

Full list of author information is

available at the end of the article

\section{照 Springer}

\begin{abstract}
In this paper, we consider a fixed point theorem that extends and unifies several existing results in the literature. We apply the proven fixed point results on the existence of solution of ordinary boundary value problems and fractional boundary value problems with integral type boundary conditions in the frame of some Caputo type fractional operators.
\end{abstract}

MSC: 47H10; 54H25; $11 \mathrm{~J} 83$

Keywords: Fractional differential equations; ODE; Generalized $\alpha$ - $h$ - $\vartheta$-contractions; Weakly contractive mappings

\section{Introduction and preliminaries}

In the last decades, two topics have been densely studied: "fixed point theory" and "fractional differential/integral equations". Relatively, fractional calculus and fractional differential/integral equations are very fresh topics for the researchers and, recently, several significant results have been recorded [1-3]. On the other hand, since the outstanding fixed point result of Banach [4], a substantial number of papers have been reported. Among them, we underline the eminent works of Geraghty [5], Boyd and Wong [6], Jaggi [7], Rhoades [8], and Dass and Gupta [9] which are essential for the main fixed point theorems.

For a non-empty set $X$ equipped with a metric $d$, we introduce a family of auxiliary functions $h: X \times X \rightarrow[0,1)$ such that

$$
\lim _{n \rightarrow \infty} h\left(\varkappa_{n}, \zeta_{n}\right)=1 \Longrightarrow \lim _{n \rightarrow \infty} d\left(\varkappa_{n}, \zeta_{n}\right)=0
$$

for all sequences $\left\{\varkappa_{n}\right\}$ and $\left\{\zeta_{n}\right\}$ in $X$ that the sequence $\left\{d\left(\varkappa_{n}, \zeta_{n}\right)\right\}$ is decreasing and convergent. The function family, defined above, is represented by $\mathcal{A}(X)$ (see, e.g., [10, 11]).

Example 1.1 Let $a_{1}, a_{2}: \mathbb{R} \times \mathbb{R} \rightarrow[0,1)$, defined by

(i) $a_{1}(\varkappa, \zeta)=k$ for some $k \in(0,1)$;

(ii) $a_{2}(\varkappa, \zeta)=\frac{t}{t+\varkappa^{2}+\zeta^{2}}$ for some $t \geq 0$.

Then $a_{1}, a_{2} \in \mathcal{A}(\mathbb{R})$.

(c) The Author(s) 2019. This article is distributed under the terms of the Creative Commons Attribution 4.0 International License (http://creativecommons.org/licenses/by/4.0/), which permits unrestricted use, distribution, and reproduction in any medium, provided you give appropriate credit to the original author(s) and the source, provide a link to the Creative Commons license, and indicate if changes were made. 
We reserve the letter $\mathcal{G}$ for all functions $\gamma:[0, \infty) \rightarrow[0,1)$ so that

$$
\gamma\left(t_{n}\right) \rightarrow 1 \text { implies } t_{n} \rightarrow 0 .
$$

A function $\gamma \in \mathcal{G}$ is also named Geraghty function.

Example 1.2 On a metric space $(X, d)$, we define a function $a: X \times X \rightarrow[0,1)$ by

$$
a(\varkappa, \zeta)=\gamma(d(\varkappa, \zeta))
$$

where $\gamma \in \mathcal{G}$. For sequences $\left\{\varkappa_{n}\right\},\left\{\zeta_{n}\right\}$ in $X$, if $\lim _{n \rightarrow \infty} a\left(\varkappa_{n}, \zeta_{n}\right)=1$, then $\lim _{n \rightarrow \infty} \gamma\left(d\left(\varkappa_{n}\right.\right.$, $\left.\left.\zeta_{n}\right)\right)=1$. Thus

$$
\lim _{n \rightarrow \infty} d\left(\varkappa_{n}, \zeta_{n}\right)=0
$$

This implies that $a \in \mathcal{A}(X)$.

By a letter $\Phi$, we present a family of auxiliary functions $\vartheta$ from $[0, \infty)$ to $[0, \infty)$ that are continuous and non-decreasing such that

$$
\vartheta(p)=0 \quad \text { if and only if } \quad p=0 \text {. }
$$

From now on, a triplet $(X, d, T)$ represents a structure:

1. A non-empty set $X$;

2. A metric on $d$ so that $(X, d)$ is complete;

3. $T$ is a self-mapping on $X$.

We also define the following inequalities on a triplet $(X, d, T)$ :

$\left(I_{1}\right) \vartheta(d(T \varkappa, T \zeta)) \leq \vartheta(d(\varkappa, \zeta))-\psi(d(\varkappa, \zeta))$ for each $\varkappa, \zeta \in X$, where $\vartheta, \psi \in \Phi$;

$\left(I_{2}\right) d(T \varkappa, T \zeta) \leq \gamma(d(\varkappa, \zeta)) d(\varkappa, \zeta)$ for each $\varkappa, \zeta \in X$, where $\gamma \in \mathcal{G}$.

Theorem 1.3 (Dutta and Choudhury [12]) On $(X, d, T)$, if $\left(I_{1}\right)$ is fulfilled, then $T$ has a fixed point.

Theorem 1.4 (Geraghty [5]) On $(X, d, T)$, if $\left(I_{2}\right)$ is fulfilled, then $T$ has a fixed point.

Popescu [13] recommended a modification on (triangular-) $\alpha$-admissible mappings, defined in $[14,15]$, as follows.

Definition 1.5 ([13]) On a triplet $(X, d, T)$, for a mapping $\theta: X \times X \rightarrow[0, \infty), T$ is called $\theta$-orbital admissible if

$$
\theta(p, T p) \geq 1 \quad \Rightarrow \quad \theta\left(T p, T^{2} p\right) \geq 1 .
$$

In addition, if the inequality

$$
\theta(p, q) \geq 1 \quad \text { and } \quad \theta(q, T q) \geq 1 \quad \Rightarrow \quad \theta(p, T q) \geq 1
$$

is fulfilled, then the $\theta$-admissible mapping $T$ is named triangular $\theta$-orbital admissible. 
Notice that each $\theta$-admissible mapping is $\theta$-orbital admissible. For more details and counter examples, see e.g. [13-17].

In addition to the triplet $(X, d, T)$ structure, if there is a mapping $\theta: X \times X \rightarrow[0, \infty)$, we represent it by a quadruplet $(X, d, T, \theta)$. It is clear that $(X, d, T, \theta)$ reduces to $(X, d, T)$ in case of $\theta(p, q)=1$ for all $p, q \in X$.

Definition 1.6 ([17]) On a structure $(X, d, T, \theta)$, we say that $X$ is $\theta$-regular if the following condition is satisfied:

( $\theta$-Regular) If $\left\{\varkappa_{n}\right\}$ is a sequence in $X$ such that $\theta\left(\varkappa_{n}, \varkappa_{n+1}\right) \geq 1$ for all $n \in \mathbb{N}$ and $\varkappa_{n} \rightarrow$ $\varkappa \in X$ as $n \rightarrow \infty$, then there exists a subsequence $\left\{\varkappa_{n_{k}}\right\}$ of $\left\{\varkappa_{n}\right\}$ such that $\theta\left(\varkappa_{n_{k}}, \varkappa\right) \geq 1$ for all $k$.

In the meantime, the fractional calculus which extends the integer order integration and differentiation to any order is one of the swiftly growing areas of research as a consequence of the results obtained when the fractional operators were utilized in modeling [18-21].

For better understanding of some real world problems, some researchers suggested recently discovered fractional operators. Among these operators, we mention the ones considered in [22-26].

On the other hand, by using fixed point theorems, the existence and uniqueness of solutions to differential/integral equations involving fractional operators were studied by a huge number of researchers. With respect to this issue, we refer to [26-35] and the references cited in those articles.

In this paper, we aim to establish some new fixed point theorems and apply the obtained results to show the existence and uniqueness of solutions to some fractional and integer order differential equations.

\section{Main results}

In this section, inspired by Geraghty contraction, we shall define a new class of mappings and investigate fixed point criteria for such mappings.

Definition 2.1 On a quadruplet $(X, d, T, \theta)$, we define the following inequalities:

$\left(I_{3}\right) \theta(\varkappa, \zeta) \vartheta(d(T \varkappa, T \zeta)) \leq h(\varkappa, \zeta) \vartheta(R(\varkappa, \zeta))$ for all $\varkappa, \zeta \in X$,

$\left(I_{4}\right) \theta(\varkappa, \zeta) \vartheta(d(T \varkappa, T \zeta)) \leq h(\varkappa, \zeta) \vartheta(J(\varkappa, \zeta))$ for all $\varkappa, \zeta \in X$,

(I5) $\theta(\varkappa, \zeta) \vartheta(d(T \varkappa, T \zeta)) \leq h(\varkappa, \zeta) \vartheta(M(\varkappa, \zeta))$ for all $\varkappa, \zeta \in X$,

where $h \in \mathcal{A}(X)$ and $\vartheta \in \Phi$, and

$$
\begin{aligned}
R(\varkappa, \zeta)= & \max \left\{\frac{d(\varkappa, T \varkappa) d(\zeta, T \zeta)}{d(\varkappa, \zeta)}, d(\varkappa, \zeta), d(\varkappa, T \varkappa),\right. \\
& \left.d(\zeta, T \zeta), \frac{d(\varkappa, T \zeta)+d(\zeta, T \varkappa)}{2}\right\}, \\
J(\varkappa, \zeta)= & \max \left\{\frac{d(\varkappa, T \varkappa) d(\zeta, T \zeta)}{d(\varkappa, \zeta)}, d(\varkappa, \zeta)\right\}, \\
M(\varkappa, \zeta)= & \max \{d(\varkappa, \zeta), d(\varkappa, T \varkappa), d(\zeta, T \zeta)\} .
\end{aligned}
$$

We say that $T$ is Jaggi type $\theta-h-\vartheta$-contraction (respectively, generalized Jaggi type $\theta-h$ $\vartheta$-contraction) if $\left(I_{3}\right)$ (respectively, $\left.\left(I_{4}\right)\right)$ is satisfied. A mapping $T$ will be named $\theta-h-\vartheta$ contraction if $\left(I_{5}\right)$ is fulfilled. 
Now, we prove the following theorem that extends and generalizes some known fixed point results.

Theorem 2.2 On a quadruplet $(X, d, T, \theta)$, if the following assumptions hold:

(i) inequality $\left(I_{3}\right)$ holds;

(ii) $T$ is continuous and forms a triangular $\theta$-orbital admissible;

(iii) there exists $\varkappa_{0} \in X$ such that $\theta\left(\varkappa_{0}, T \varkappa_{0}\right) \geq 1$;

then $T$ has a fixed point.

Proof On account of (iii), there is $\varkappa_{0} \in X$ with

$$
\theta\left(\varkappa_{0}, T \varkappa_{0}\right) \geq 1
$$

Define an iterative sequence $\left\{\varkappa_{n}\right\}$ by $\varkappa_{n}=T \varkappa_{n-1}$ for all $n \in \mathbb{N}$. Suppose that for some positive integer $k$, we have $\varkappa_{k}=\varkappa_{k+1}$. This implies that $T \varkappa_{k}=\varkappa_{k+1}=\varkappa_{k}$, that is, $\varkappa_{k}$ is a fixed point of $T$. Thus, we shall assume that $\varkappa_{n} \neq \varkappa_{n+1}$ for all $n=0,1,2, \ldots$

On account of inequality $\left(I_{3}\right)$, for all $n \in \mathbb{N}$, we have

$$
\begin{aligned}
\vartheta\left(d\left(\varkappa_{n}, \varkappa_{n+1}\right)\right) & \leq \theta\left(\varkappa_{n-1}, \varkappa_{n}\right) \vartheta\left(d\left(\varkappa_{n}, \varkappa_{n+1}\right)\right) \\
& =\theta\left(\varkappa_{n-1}, \varkappa_{n}\right) \vartheta\left(d\left(T \varkappa_{n-1}, T \varkappa_{n}\right)\right) \\
& \leq h\left(\varkappa_{n-1}, \varkappa_{n}\right) \vartheta\left(R\left(\varkappa_{n-1}, \varkappa_{n}\right)\right) \\
& <\vartheta\left(R\left(\varkappa_{n-1}, \varkappa_{n}\right)\right) .
\end{aligned}
$$

On the other hand,

$$
\begin{aligned}
R\left(\varkappa_{n-1}, \varkappa_{n}\right)= & \max \left\{\frac{d\left(\varkappa_{n-1}, T \varkappa_{n-1}\right) d\left(\varkappa_{n}, T \varkappa_{n}\right)}{d\left(\varkappa_{n-1}, \varkappa_{n}\right)}, d\left(\varkappa_{n-1}, \varkappa_{n}\right), d\left(\varkappa_{n-1}, T \varkappa_{n-1}\right),\right. \\
& \left.d\left(\varkappa_{n}, T \varkappa_{n}\right), \frac{d\left(\varkappa_{n-1}, T \varkappa_{n}\right)+d\left(\varkappa_{n}, T \varkappa_{n-1}\right)}{2}\right\} \\
= & \max \left\{d\left(\varkappa_{n}, \varkappa_{n+1}\right), d\left(\varkappa_{n-1}, \varkappa_{n}\right), d\left(\varkappa_{n-1}, \varkappa_{n}\right), d\left(\varkappa_{n}, \varkappa_{n+1}\right),\right. \\
& \left.\frac{d\left(\varkappa_{n-1}, \varkappa_{n+1}\right)+d\left(\varkappa_{n}, \varkappa_{n}\right)}{2}\right\} \\
= & \max \left\{d\left(\varkappa_{n-1}, \varkappa_{n}\right), d\left(\varkappa_{n}, \varkappa_{n+1}\right), \frac{d\left(\varkappa_{n-1}, \varkappa_{n+1}\right)}{2}\right\} \\
\leq & \max \left\{d\left(\varkappa_{n-1}, \varkappa_{n}\right), d\left(\varkappa_{n}, \varkappa_{n+1}\right), \frac{d\left(\varkappa_{n-1}, \varkappa_{n}\right)+d\left(\varkappa_{n}, \varkappa_{n+1}\right)}{2}\right\} \\
= & \max \left\{d\left(\varkappa_{n-1}, \varkappa_{n}\right), d\left(\varkappa_{n}, \varkappa_{n+1}\right)\right\} .
\end{aligned}
$$

If $R\left(\varkappa_{n-1}, \varkappa_{n}\right)=d\left(\varkappa_{n}, \varkappa_{n+1}\right)$, employing (2.4), we conclude that

$$
\begin{aligned}
\vartheta\left(d\left(\varkappa_{n}, \varkappa_{n+1}\right)\right) & <\vartheta\left(R\left(\varkappa_{n-1}, \varkappa_{n}\right)\right) \\
& =\vartheta\left(d\left(\varkappa_{n}, \varkappa_{n+1}\right)\right),
\end{aligned}
$$


a contradiction. So, we conclude that, for all $n \in \mathbb{N}$,

$$
R\left(\varkappa_{n-1}, \varkappa_{n}\right)=d\left(\varkappa_{n-1}, \varkappa_{n}\right) .
$$

Now, from (2.4) and (2.5), we get that

$$
\vartheta\left(d\left(\varkappa_{n}, \varkappa_{n+1}\right)\right)<\vartheta\left(d\left(\varkappa_{n-1}, \varkappa_{n}\right)\right) .
$$

Using the monotony of $\vartheta$ implies that, for all $n \in \mathbb{N}$,

$$
d\left(\varkappa_{n}, \varkappa_{n+1}\right)<d\left(\varkappa_{n-1}, \varkappa_{n}\right) .
$$

So, the sequence $\left\{d\left(\varkappa_{n}, \varkappa_{n+1}\right)\right\}$ is non-negative and decreasing. Eventually, there exists $r \geq$ 0 such that $\lim _{n \rightarrow \infty} d\left(\varkappa_{n}, \varkappa_{n+1}\right)=r$. Thereafter, we illustrate that $r=0$. Suppose, on the contrary, that $r>0$. Then, from (2.4) and (2.5), we have

$$
0<\frac{\vartheta\left(d\left(\varkappa_{n}, \varkappa_{n+1}\right)\right)}{\vartheta\left(d\left(\varkappa_{n-1}, \varkappa_{n}\right)\right)} \leq h\left(\varkappa_{n-1}, \varkappa_{n}\right)
$$

which implies that $\lim _{n \rightarrow \infty} h\left(\varkappa_{n-1}, \varkappa_{n}\right)=1$. Since $h \in \mathcal{A}$,

$$
\lim _{n \rightarrow \infty} d\left(\varkappa_{n-1}, \varkappa_{n}\right)=0 .
$$

It yields $r=0$ that is a contradiction. Hereby,

$$
\lim _{n \rightarrow \infty} d\left(\varkappa_{n}, \varkappa_{n+1}\right)=0
$$

As the next step, we indicate that the sequence $\left\{\varkappa_{n}\right\}$ is fundamental (Cauchy). Suppose, on the contrary, that the iterative sequence $\left\{\varkappa_{n}\right\}$ is not fundamental. Then there exists $\epsilon>0$ such that, for all $k \in \mathbb{N}$, we can find $m_{k} \geq n_{k}>k$ such that

$$
d\left(\varkappa_{n_{k}}, \varkappa_{m_{k}}\right) \geq \epsilon .
$$

In addition, it may be assumed that

$$
d\left(\varkappa_{n_{k}}, \varkappa_{m_{k}-1}\right)<\epsilon
$$

by choosing $m_{k}$ as small as possible. Accordingly, for each $k \in \mathbb{N}$, we find

$$
\begin{aligned}
\epsilon & \leq d\left(\varkappa_{n_{k}}, \varkappa_{m_{k}}\right) \leq d\left(\varkappa_{n_{k}}, \varkappa_{m_{k}-1}\right)+d\left(\varkappa_{m_{k}-1}, \varkappa_{m_{k}}\right) \\
& \leq \epsilon+d\left(\varkappa_{m_{k}-1}, \varkappa_{m_{k}}\right) .
\end{aligned}
$$

Letting $k \rightarrow \infty$, we get

$$
\lim _{n \rightarrow \infty} d\left(\varkappa_{n_{k}}, \varkappa_{m_{k}}\right)=\epsilon .
$$


Note that, for any $k \in \mathbb{N}$,

$$
\begin{aligned}
\vartheta\left(d\left(\varkappa_{n_{k}+1}, \varkappa_{m_{k}+1}\right)\right) & \leq \theta\left(\varkappa_{n_{k}}, \varkappa_{m_{k}}\right) \vartheta\left(d\left(\varkappa_{n_{k}+1}, \varkappa_{m_{k}+1}\right)\right) \\
& =\theta\left(\varkappa_{n_{k}}, \varkappa_{m_{k}}\right) \vartheta\left(d\left(T \varkappa_{n_{k}}, T \varkappa_{m_{k}}\right)\right) \\
& \leq h\left(\varkappa_{n_{k}}, \varkappa_{m_{k}}\right) \vartheta\left(R\left(\varkappa_{n_{k}}, \varkappa_{m_{k}}\right)\right) .
\end{aligned}
$$

Also, for any $k \in \mathbb{N}$, we have

$$
\begin{aligned}
R\left(\varkappa_{n_{k}}, \varkappa_{m_{k}}\right)= & \max \left\{\frac{d\left(\varkappa_{n_{k}}, T \varkappa_{n_{k}}\right) d\left(\varkappa_{m_{k}}, T \varkappa_{m_{k}}\right)}{d\left(\varkappa_{n_{k}}, \varkappa_{m_{k}}\right)}, d\left(\varkappa_{n_{k}}, \varkappa_{m_{k}}\right), d\left(\varkappa_{n_{k}}, T \varkappa_{n_{k}}\right),\right. \\
& \left.d\left(\varkappa_{m_{k}}, T \varkappa_{m_{k}}\right), \frac{d\left(\varkappa_{n_{k}}, T \varkappa_{m_{k}}\right)+d\left(\varkappa_{m_{k}}, T \varkappa_{n_{k}}\right)}{2}\right\} \\
= & \max \left\{\frac{d\left(\varkappa_{n_{k}}, \varkappa_{n_{k}+1}\right) d\left(\varkappa_{m_{k}}, \varkappa_{m_{k}+1}\right)}{d\left(\varkappa_{n_{k}}, \varkappa_{m_{k}}\right)}, d\left(\varkappa_{k}, \varkappa_{m_{k}}\right), d\left(\varkappa_{n_{k}}, \varkappa_{n_{k}+1}\right),\right. \\
& \left.d\left(\varkappa_{m_{k}}, \varkappa_{m_{k}+1}\right), \frac{d\left(\varkappa_{n_{k}}, \varkappa_{m_{k}+1}\right)+d\left(\varkappa_{m_{k}}, \varkappa_{n_{k}+1}\right)}{2}\right\} \\
\leq & \max \left\{\frac{d\left(\varkappa_{n_{k}}, \varkappa_{n_{k}+1}\right) d\left(\varkappa_{m_{k}}, \varkappa_{m_{k}+1}\right)}{d\left(\varkappa_{n_{k}}, \varkappa_{m_{k}}\right)}, d\left(\varkappa_{n_{k}}, \varkappa_{m_{k}}\right), d\left(\varkappa_{n_{k}}, \varkappa_{n_{k}+1}\right),\right. \\
& d\left(\varkappa_{m_{k}}, \varkappa_{m_{k}+1}\right), \\
& \left.\frac{d\left(\varkappa_{n_{k}}, \varkappa_{m_{k}}\right)+d\left(\varkappa_{m_{k}}, \varkappa_{m_{k}+1}\right)+d\left(\varkappa_{m_{k}}, \varkappa_{n_{k}}\right)+d\left(\varkappa_{n_{k}}, \varkappa_{n_{k}+1}\right)}{2}\right\} .
\end{aligned}
$$

Keeping $\lim _{k \rightarrow \infty} d\left(\varkappa_{n_{k}}, \varkappa_{n_{k}+1}\right)=0$ in mind, the above inequality yields that

$$
\lim _{k \rightarrow \infty} R\left(\varkappa_{n_{k}}, \varkappa_{m_{k}}\right)=\lim _{k \rightarrow \infty} d\left(\varkappa_{n_{k}}, \varkappa_{m_{k}}\right) .
$$

On account of the triangular inequality and letting $k \rightarrow \infty$, we derive

$$
\begin{aligned}
\lim _{k \rightarrow \infty} d\left(\varkappa_{n_{k}}, \varkappa_{m_{k}}\right) & \leq \lim _{k \rightarrow \infty}\left(d\left(\varkappa_{n_{k}}, \varkappa_{n_{k}+1}\right)+d\left(\varkappa_{n_{k}+1}, \varkappa_{m_{k}+1}\right)+d\left(\varkappa_{m_{k}+1}, \varkappa_{m_{k}}\right)\right) \\
& =\lim _{k \rightarrow \infty} d\left(\varkappa_{n_{k}+1}, \varkappa_{m_{k}+1}\right) .
\end{aligned}
$$

Keeping the continuity of $\vartheta$ in mind and combining (2.6), (2.7), and (2.8), we get

$$
\lim _{k \rightarrow \infty} \vartheta\left(d\left(\varkappa_{n_{k}}, \varkappa_{m_{k}}\right)\right) \leq \lim _{k \rightarrow \infty} h\left(\varkappa_{n_{k}}, \varkappa_{m_{k}}\right) \lim _{k \rightarrow \infty} \vartheta\left(d\left(\varkappa_{n_{k}}, \varkappa_{m_{k}}\right)\right)
$$

Since $\lim _{k \rightarrow \infty} d\left(\varkappa_{n_{k}}, \varkappa_{m_{k}}\right)=\epsilon>0$, we deduce that

$$
\lim _{k \rightarrow \infty} h\left(\varkappa_{n_{k}}, \varkappa_{m_{k}}\right)=1
$$

Since $h \in \mathcal{A}(X)$, then

$$
\lim _{k \rightarrow \infty} d\left(\varkappa_{n_{k}}, \varkappa_{m_{k}}\right)=0
$$


It is a contradiction. As a result, the iterative sequence $\left\{\varkappa_{n}\right\}$ is Cauchy. Consequently, there exists $\varkappa^{*} \in X$ such that $\lim _{n \rightarrow \infty} \varkappa_{n}=\varkappa^{*}$. Regarding the continuity of $T$, we find

$$
\lim _{n \rightarrow \infty} \varkappa_{n+1}=\lim _{n \rightarrow \infty} T \varkappa_{n}=T \varkappa^{*}
$$

Thus $T \varkappa^{*}=\varkappa^{*}$.

Definition 2.3 On a structure $(X, d, T, \theta)$, we consider the following inequality:

(I $\left.\sigma_{6}\right) \theta(\varkappa, \zeta) \vartheta(d(T \varkappa, T \zeta)) \leq h(\varkappa, \zeta) \vartheta(Q(\varkappa, \zeta))$ for all $\varkappa, \zeta \in X$,

where $h \in \mathcal{A}(X), \vartheta \in \Phi$, and

$$
Q(\varkappa, \zeta)=\max \left\{\frac{d(\varkappa, T \varkappa)[1+d(\eta, T \eta)]}{1+d(\varkappa, \zeta)}, \frac{d(\zeta, T \zeta)[1+d(\varkappa, T \varkappa)]}{1+d(\varkappa, \zeta)}, d(\varkappa, \zeta)\right\} .
$$

A self-mapping $T$ is called a generalized Dass-Gupta type $\theta-h-\vartheta$-contraction if $\left(I_{4}\right)$ is satisfied.

Theorem 2.4 On $(X, d, T, \theta)$, we assume that

(i) inequality $\left(I_{6}\right)$ holds;

(ii) $T$ is continuous and triangular $\theta$-orbital admissible;

(iii) there exists $\varkappa_{0} \in X$ such that $\theta\left(\varkappa_{0}, T \varkappa_{0}\right) \geq 1$.

Then $T$ has a fixed point.

Proof From condition (iii), there exists $\varkappa_{0} \in X$ such that

$$
\theta\left(\varkappa_{0}, T \varkappa_{0}\right) \geq 1
$$

Define the sequence $\left\{\varkappa_{n}\right\}$ by $\varkappa_{n}=T \varkappa_{n-1}$ for all $n \in \mathbb{N}$. Suppose that, for some positive integer $k$, we have $\varkappa_{k}=\varkappa_{k+1}$. This implies that $T \varkappa_{k}=\varkappa_{k+1}=\varkappa_{k}$, that is, $\varkappa_{k}$ is a fixed point of $T$. So, we can assume that $\varkappa_{n} \neq \varkappa_{n+1}, n=0,1,2, \ldots$.

Taking inequality $\left(I_{6}\right)$ into account, we find

$$
\begin{aligned}
\vartheta\left(d\left(\varkappa_{n}, \varkappa_{n+1}\right)\right) & \leq \theta\left(\varkappa_{n-1}, \varkappa_{n}\right) \vartheta\left(d\left(\varkappa_{n}, \varkappa_{n+1}\right)\right) \\
& =\theta\left(\varkappa_{n-1}, \varkappa_{n}\right) \vartheta\left(d\left(T \varkappa_{n-1}, T \varkappa_{n}\right)\right) \\
& \leq h\left(\varkappa_{n-1}, \varkappa_{n}\right) \vartheta\left(Q\left(\varkappa_{n-1}, \varkappa_{n}\right)\right) \\
& <\vartheta\left(Q\left(\varkappa_{n-1}, \varkappa_{n}\right)\right) .
\end{aligned}
$$

On the other hand,

$$
\begin{aligned}
Q\left(\varkappa_{n-1}, \varkappa_{n}\right)= & \max \left\{\frac{d\left(\varkappa_{n-1}, T \varkappa_{n-1}\right)\left[1+d\left(\varkappa_{n}, T \varkappa_{n}\right)\right]}{1+d\left(\varkappa_{n-1}, \varkappa_{n}\right)}, \frac{d\left(\varkappa_{n}, T \varkappa_{n}\right)\left[1+d\left(\varkappa_{n-1}, T \varkappa_{n-1}\right)\right]}{1+d\left(\varkappa_{n-1}, \varkappa_{n}\right)},\right. \\
& \left.d\left(\varkappa_{n-1}, \varkappa_{n}\right)\right\} \\
= & \max \left\{\frac{d\left(\varkappa_{n-1}, \varkappa_{n}\right)\left[1+d\left(\varkappa_{n}, \varkappa_{n+1}\right)\right]}{1+d\left(\varkappa_{n-1}, \varkappa_{n}\right)}, \frac{d\left(\varkappa_{n}, \varkappa_{n+1}\right)\left[1+d\left(\varkappa_{n-1}, \varkappa_{n}\right)\right]}{1+d\left(\varkappa_{n-1}, \varkappa_{n}\right)},\right.
\end{aligned}
$$




$$
\begin{aligned}
& \left.d\left(\varkappa_{n-1}, \varkappa_{n}\right)\right\} \\
= & \max \left\{\frac{d\left(\varkappa_{n-1}, \varkappa_{n}\right)\left[1+d\left(\varkappa_{n}, \varkappa_{n+1}\right)\right]}{1+d\left(\varkappa_{n-1}, \varkappa_{n}\right)}, d\left(\varkappa_{n}, \varkappa_{n+1}\right), d\left(\varkappa_{n-1}, \varkappa_{n}\right)\right\} .
\end{aligned}
$$

If $d\left(\varkappa_{n-1}, \varkappa_{n}\right) \leq d\left(\varkappa_{n}, \varkappa_{n+1}\right)$, then $Q\left(\varkappa_{n-1}, \varkappa_{n}\right)=d\left(\varkappa_{n}, \varkappa_{n+1}\right)$, applying (2.4), we deduce that

$$
\begin{aligned}
\vartheta\left(d\left(\varkappa_{n}, \varkappa_{n+1}\right)\right) & <\vartheta\left(Q\left(\varkappa_{n-1}, \varkappa_{n}\right)\right) \\
& =\vartheta\left(d\left(\varkappa_{n}, \varkappa_{n+1}\right)\right),
\end{aligned}
$$

which yields a contradiction. Accordingly, we find

$$
Q\left(\varkappa_{n-1}, \varkappa_{n}\right)=d\left(\varkappa_{n-1}, \varkappa_{n}\right)
$$

for all $n \in \mathbb{N}$. On account of (2.4) and (2.5), we derive that

$$
\vartheta\left(d\left(\varkappa_{n}, \varkappa_{n+1}\right)\right)<\vartheta\left(d\left(\varkappa_{n-1}, \varkappa_{n}\right)\right) .
$$

Employing the monotonicity of $\vartheta$, we get

$$
d\left(\varkappa_{n}, \varkappa_{n+1}\right)<d\left(\varkappa_{n-1}, \varkappa_{n}\right)
$$

for all $n \in \mathbb{N}$. As a result, we deduce that $\left\{d\left(\varkappa_{n}, \varkappa_{n+1}\right)\right\}$ is a non-negative and decreasing sequence. As an immediate consequence, we conclude that there exists $r \geq 0$ such that $\lim _{n \rightarrow \infty} d\left(\varkappa_{n}, \varkappa_{n+1}\right)=r$. In what follows, we assert that the limit $r=0$. Suppose, on the contrary, that $r>0$. Then, from (2.4) and (2.5), we have

$$
0<\frac{\vartheta\left(d\left(\varkappa_{n}, \varkappa_{n+1}\right)\right)}{\vartheta\left(d\left(\varkappa_{n-1}, \varkappa_{n}\right)\right)} \leq h\left(\varkappa_{n-1}, \varkappa_{n}\right)
$$

which implies that $\lim _{n \rightarrow \infty} h\left(\varkappa_{n-1}, \varkappa_{n}\right)=1$. Since $h \in \mathcal{A}(X)$,

$$
\lim _{n \rightarrow \infty} d\left(\varkappa_{n-1}, \varkappa_{n}\right)=0=r
$$

It contradicts our assumption. Therefore

$$
r=\lim _{n \rightarrow \infty} d\left(\varkappa_{n}, \varkappa_{n+1}\right)=0 .
$$

In the sequel, we shall affirm that the sequence $\left\{\varkappa_{n}\right\}$ is fundamental (Cauchy) sequence. Suppose, on the contrary, that $\left\{\varkappa_{n}\right\}$ is not a fundamental sequence. Accordingly, there exists $\epsilon>0$ such that, for all $k \in \mathbb{N}$, we can find $m_{k} \geq n_{k}>k$ such that

$$
d\left(\varkappa_{n_{k}}, \varkappa_{m_{k}}\right) \geq \epsilon
$$

Also, choosing $m_{k}$ as small as possible, it may be assumed that

$$
d\left(\varkappa_{n_{k}}, \varkappa_{m_{k}-1}\right)<\epsilon
$$


Accordingly, for each $k \in \mathbb{N}$, we have

$$
\begin{aligned}
\epsilon & \leq d\left(\varkappa_{n_{k}}, \varkappa_{m_{k}}\right) \leq d\left(\varkappa_{n_{k}}, \varkappa_{m_{k}-1}\right)+d\left(\varkappa_{m_{k}-1}, \varkappa_{m_{k}}\right) \\
& \leq \epsilon+d\left(\varkappa_{m_{k}-1}, \varkappa_{m_{k}}\right) .
\end{aligned}
$$

As $k \rightarrow \infty$ in the inequality above, we get

$$
\lim _{n \rightarrow \infty} d\left(\varkappa_{n_{k}}, \varkappa_{m_{k}}\right)=\epsilon
$$

Note that, for any $k \in \mathbb{N}$,

$$
\begin{aligned}
\vartheta\left(d\left(\varkappa_{n_{k}+1}, \varkappa_{m_{k}+1}\right)\right) & \leq \theta\left(\varkappa_{n_{k}}, \varkappa_{m_{k}}\right) \vartheta\left(d\left(\varkappa_{n_{k}+1}, \varkappa_{m_{k}+1}\right)\right) \\
& =\theta\left(\varkappa_{n_{k}}, \varkappa_{m_{k}}\right) \vartheta\left(d\left(T \varkappa_{n_{k}}, T \varkappa_{m_{k}}\right)\right) \\
& \leq h\left(\varkappa_{n_{k}}, \varkappa_{m_{k}}\right) \vartheta\left(Q\left(\varkappa_{n_{k}}, \varkappa_{m_{k}}\right)\right) .
\end{aligned}
$$

Also, for any $k \in \mathbb{N}$, we have

$$
\begin{aligned}
Q\left(\varkappa_{n_{k}}, \varkappa_{m_{k}}\right)= & \max \left\{\frac{d\left(\varkappa_{n_{k}}, T \varkappa_{n_{k}}\right)\left[1+d\left(\varkappa_{m_{k}}, T \varkappa_{m_{k}}\right)\right]}{1+d\left(\varkappa_{n_{k}}, \varkappa_{m_{k}}\right)},\right. \\
& \left.\frac{d\left(\varkappa_{m_{k}}, T \varkappa_{m_{k}}\right)\left[1+d\left(\varkappa_{n_{k}}, T \varkappa_{n_{k}}\right)\right]}{1+d\left(\varkappa_{n_{k}}, \varkappa_{m_{k}}\right)}, d\left(\varkappa_{n_{k}}, \varkappa_{m_{k}}\right)\right\} \\
= & \max \left\{\frac{d\left(\varkappa_{n_{k}}, \varkappa_{n_{k}+1}\right)\left[1+d\left(\varkappa_{m_{k}}, \varkappa_{m_{k}+1}\right)\right]}{1+d\left(\varkappa_{n_{k}}, \varkappa_{m_{k}}\right)},\right. \\
& \left.\frac{d\left(\varkappa_{m_{k}}, \varkappa_{m_{k}+1}\right)\left[1+d\left(\varkappa_{n_{k}}, \varkappa_{n_{k}+1}\right)\right]}{1+d\left(\varkappa_{n_{k}}, \varkappa_{m_{k}}\right)}, d\left(\varkappa_{n_{k}}, \varkappa_{m_{k}}\right)\right\} .
\end{aligned}
$$

On the other hand, on account of the observation $\lim _{k \rightarrow \infty} d\left(\varkappa_{n_{k}}, \varkappa_{n_{k}+1}\right)=0$, we find

$$
\lim _{k \rightarrow \infty} Q\left(\varkappa_{n_{k}}, \varkappa_{m_{k}}\right)=\lim _{k \rightarrow \infty} d\left(\varkappa_{n_{k}}, \varkappa_{m_{k}}\right) .
$$

On account of the triangular inequality and taking the limit as $n \rightarrow \infty$, we derive

$$
\begin{aligned}
\lim _{k \rightarrow \infty} d\left(\varkappa_{n_{k}}, \varkappa_{m_{k}}\right) & \leq \lim _{k \rightarrow \infty}\left(d\left(\varkappa_{n_{k}}, \varkappa_{n_{k}+1}\right)+d\left(\varkappa_{n_{k}+1}, \varkappa_{m_{k}+1}\right)+d\left(\varkappa_{m_{k}+1}, \varkappa_{m_{k}}\right)\right) \\
& =\lim _{k \rightarrow \infty} d\left(\varkappa_{n_{k}+1}, \varkappa_{m_{k}+1}\right) .
\end{aligned}
$$

Combining (2.12), (2.13), and (2.14) with the continuity of $\vartheta$, we conclude that

$$
\lim _{k \rightarrow \infty} \vartheta\left(d\left(\varkappa_{n_{k}}, \varkappa_{m_{k}}\right)\right) \leq \lim _{k \rightarrow \infty} h\left(\varkappa_{n_{k}}, \varkappa_{m_{k}}\right) \lim _{k \rightarrow \infty} \vartheta\left(d\left(\varkappa_{n_{k}}, \varkappa_{m_{k}}\right)\right)
$$

Keeping the assumption $\lim _{k \rightarrow \infty} d\left(\varkappa_{n_{k}}, \varkappa_{m_{k}}\right)=\epsilon>0$ in mind, we deduce that

$$
\lim _{k \rightarrow \infty} h\left(\varkappa_{n_{k}}, \varkappa_{m_{k}}\right)=1
$$


Regarding that $h \in \mathcal{A}(X)$, we get

$$
\lim _{k \rightarrow \infty} d\left(\varkappa_{n_{k}}, \varkappa_{m_{k}}\right)=0
$$

a contradiction. As a result, the sequence $\left\{\varkappa_{n}\right\}$ is fundamental. Furthermore, there exists $\varkappa^{*} \in X$ such that $\lim _{n \rightarrow \infty} \varkappa_{n}=\varkappa^{*}$. Since $T$ is a continuous function, therefore

$$
\lim _{n \rightarrow \infty} \varkappa_{n+1}=\lim _{n \rightarrow \infty} T \varkappa_{n}=T \varkappa^{*}
$$

Thus $T \varkappa^{*}=\varkappa^{*}$.

Definition 2.5 On a quadruplet $(X, d, T, \theta)$, we define

$$
\left(I_{3}\right)^{*} \theta(\varkappa, \zeta) \vartheta(d(T \varkappa, T \zeta)) \leq h(\varkappa, \zeta) \vartheta(R(\varkappa, \zeta)) \text { for all } \varkappa, \zeta \in X
$$

where $h \in \mathcal{A}(X)$ and $\vartheta \in \Phi$, and $R(\varkappa, \zeta)$ is defined as in (2.1), under the condition

$$
\lim _{n \rightarrow \infty} h\left(\varkappa_{n}, \zeta_{n}\right)=1 \quad \Longrightarrow \quad \lim _{n \rightarrow \infty} d\left(T \varkappa_{n}, T \zeta_{n}\right)=0
$$

for all sequences $\left\{\varkappa_{n}\right\},\left\{\zeta_{n}\right\} \subseteq X$ that $\theta\left(\varkappa_{n}, \zeta_{n}\right) \neq 0, \forall n \in \mathbb{N}$. We say that $T$ is a generalized Jaggi type $\theta-h-\vartheta$-contraction if $\left(I_{3}\right)^{*}$ is satisfied.

Instead of the continuity condition, in Theorem 2.2, we propose that $X$ is $\theta$-regular, as follows.

Theorem 2.6 On a quadruplet $(X, d, T, \theta)$, if the following assumptions hold:

(i) inequality $\left(I_{3}\right)^{*}$ holds;

(ii) $X$ is $\theta$-regular and $T$ is continuous and forms triangular $\theta$-orbital admissible;

(iii) there exists $\varkappa_{0} \in X$ such that $\theta\left(\varkappa_{0}, T \varkappa_{0}\right) \geq 1$;

then $T$ has a fixed point.

Proof From condition (iii), there exists $\varkappa_{0} \in X$ such that

$$
\theta\left(\varkappa_{0}, T \varkappa_{0}\right) \geq 1
$$

Define the sequence $\left\{\varkappa_{n}\right\}$ by $\varkappa_{n}=T \varkappa_{n-1}$ for all $n \in \mathbb{N}$. Following the related lines in the proof of Theorem 2.2, we know that the sequence $\left\{\varkappa_{n}\right\}$ is convergent to some $\varkappa^{*} \in X$ and $\theta\left(\varkappa_{n}, \varkappa_{n+1}\right) \geq 1$ for all $n \in \mathbb{N}$. Since the sequence $X$ is $\theta$-regular, there exists a subsequence $\left\{\varkappa_{n_{k}}\right\}$ with $\theta\left(\varkappa_{n_{k}}, \varkappa^{*}\right) \geq 1$ for each $k \in \mathbb{N}$. Without loss of generality, we assume that

$$
\theta\left(\varkappa_{n}, \varkappa^{*}\right) \geq 1 \quad \text { for all } n \in \mathbb{N} .
$$

Applying (2.15), for all $n \in \mathbb{N}$, we get

$$
\begin{aligned}
\vartheta\left(d\left(\varkappa_{n+1}, T \varkappa^{*}\right)\right) & =\vartheta\left(d\left(T \varkappa_{n}, T \varkappa^{*}\right)\right) \\
& \leq \theta\left(\varkappa_{n}, \varkappa^{*}\right) \vartheta\left(d\left(T \varkappa_{n}, T \varkappa^{*}\right)\right) \\
& \leq h\left(\varkappa_{n}, \varkappa^{*}\right) \vartheta\left(R\left(\varkappa_{n}, \varkappa^{*}\right)\right) .
\end{aligned}
$$


Also, we have

$$
\begin{aligned}
R\left(\varkappa_{n}, \varkappa^{*}\right)= & \max \left\{\frac{d\left(\varkappa_{n}, T \varkappa_{n}\right) d\left(\varkappa^{*}, T \varkappa^{*}\right)}{d\left(\varkappa_{n}, \varkappa^{*}\right)}, d\left(\varkappa_{n}, \varkappa^{*}\right), d\left(\varkappa_{n}, T \varkappa_{n}\right), d\left(\varkappa^{*}, T \varkappa^{*}\right),\right. \\
& \left.\frac{d\left(\varkappa_{n}, T \varkappa^{*}\right)+d\left(\varkappa^{*}, T \varkappa_{n}\right)}{2}\right\} \\
= & \max \left\{\frac{d\left(\varkappa_{n}, \varkappa_{n+1}\right) d\left(\varkappa^{*}, T \varkappa^{*}\right)}{d\left(\varkappa_{n}, \varkappa^{*}\right)}, d\left(\varkappa_{n}, \varkappa^{*}\right), d\left(\varkappa_{n}, \varkappa_{n+1}\right), d\left(\varkappa^{*}, T \varkappa^{*}\right),\right. \\
& \left.\frac{d\left(\varkappa_{n}, T \varkappa^{*}\right)+d\left(\varkappa^{*}, \varkappa_{n+1}\right)}{2}\right\} .
\end{aligned}
$$

Since $\lim _{n \rightarrow \infty} d\left(\varkappa_{n}, \varkappa^{*}\right)=0$, then $\lim _{n \rightarrow \infty} R\left(\varkappa_{n}, \varkappa^{*}\right)=d\left(\varkappa^{*}, T \varkappa^{*}\right)$. Applying (2.10) and the continuity of $\vartheta$, we get $\lim _{n \rightarrow \infty} h\left(\varkappa_{n}, \varkappa^{*}\right)=1$, and so

$$
d\left(\varkappa^{*}, T \varkappa^{*}\right)=\lim _{n \rightarrow \infty} d\left(T \varkappa_{n}, T \varkappa^{*}\right)=0
$$

Therefore $T \varkappa^{*}=\varkappa^{*}$.

Definition 2.7 On a structure $(X, d, T, \theta)$, we consider the following inequality:

$\left(I_{6}\right)^{*} \theta(\varkappa, \zeta) \vartheta(d(T \varkappa, T \zeta)) \leq h(\varkappa, \zeta) \vartheta(Q(\varkappa, \zeta))$ for all $\varkappa, \zeta \in X$,

where $h \in \mathcal{A}(X), \vartheta \in \Phi$, and $Q(\varkappa, \zeta)$ is defined as in (2.9) under the condition

$$
\lim _{n \rightarrow \infty} h\left(\varkappa_{n}, \zeta_{n}\right)=1 \Longrightarrow \lim _{n \rightarrow \infty} d\left(T \varkappa_{n}, T \zeta_{n}\right)=0
$$

for all sequences $\left\{\varkappa_{n}\right\},\left\{\zeta_{n}\right\} \subseteq X$ that $\theta\left(\varkappa_{n}, \zeta_{n}\right) \neq 0, \forall n \in \mathbb{N}$.

Theorem 2.8 For $(X, d, T, \theta)$, we assume that

(i) inequality $\left(I_{6}\right)^{*}$ holds;

(ii) $X$ is $\theta$-regular and $T$ triangular $\theta$-orbital admissible;

(iii) there exists $\varkappa_{0} \in X$ such that $\theta\left(\varkappa_{0}, T \varkappa_{0}\right) \geq 1$.

Then $T$ has a fixed point.

We skipped the proof since it is verbatim of the proof of Theorem 2.6.

In what follows, we consider the uniqueness of the derived fixed point of certain mappings that are mentioned in the theorems above. Let $\operatorname{Fix}(T)=\{\varkappa \in X: T(\varkappa)=\varkappa\}$. We propose the following criteria for the uniqueness:

$\left(\mathrm{H}_{0}\right)$ For all $p, q \in \operatorname{Fix}(T)$, we have $\theta(p, q) \geq 1$.

Theorem 2.9 In addition to the assumptions of Theorem 2.2 (resp. Theorem 2.6), if $\left(\mathrm{H}_{1}\right)$ holds, then the obtained fixed point in Theorem 2.2 (resp. Theorem 2.6) is unique.

Proof Let $\varkappa^{*}, \zeta^{*} \in X$ be two distinct fixed points of $T$, that is, $\varkappa^{*} \neq \zeta^{*}$. Due to $\left(\mathrm{H}_{0}\right)$, we have $\theta\left(\varkappa^{*}, \zeta^{*}\right) \geq 1$. Herewith

$$
\begin{aligned}
\vartheta\left(d\left(\varkappa^{*}, \zeta^{*}\right)\right) & =\vartheta\left(d\left(T \varkappa^{*}, T \zeta^{*}\right)\right) \\
& \leq \theta\left(\varkappa^{*}, \zeta^{*}\right) \vartheta\left(d\left(T \varkappa^{*}, T \zeta^{*}\right)\right)
\end{aligned}
$$




$$
\begin{aligned}
& \leq h\left(\varkappa^{*}, \zeta^{*}\right) \vartheta\left(R\left(\varkappa^{*}, \zeta^{*}\right)\right) \\
& <\vartheta\left(R\left(\varkappa^{*}, \zeta^{*}\right)\right) .
\end{aligned}
$$

Besides, we have

$$
\begin{aligned}
R\left(\varkappa^{*}, \zeta^{*}\right)= & \max \left\{\frac{d\left(\varkappa^{*}, T \varkappa^{*}\right) d\left(\zeta^{*}, T \zeta^{*}\right)}{d\left(\varkappa^{*}, \zeta^{*}\right)}, d\left(\varkappa^{*}, \zeta^{*}\right), d\left(\varkappa^{*}, T \varkappa^{*}\right), d\left(\zeta^{*}, T \zeta^{*}\right),\right. \\
& \left.\frac{d\left(\varkappa^{*}, T \zeta^{*}\right)+d\left(\zeta^{*}, T \varkappa^{*}\right)}{2}\right\} . \\
= & d\left(\varkappa^{*}, \zeta^{*}\right) .
\end{aligned}
$$

Combining (2.17) and (2.18), we have $\vartheta\left(d\left(\varkappa^{*}, \zeta^{*}\right)\right)<\vartheta\left(d\left(\varkappa^{*}, \zeta^{*}\right)\right)$, a contradiction. Eventually, it implies that $\varkappa^{*}=\zeta^{*}$.

Theorem 2.10 In addition to the assumptions of Theorem 2.4 (resp. Theorem 2.8), if $\left(\mathrm{H}_{0}\right)$ holds, then the obtained fixed point in Theorem 2.4 (resp. Theorem 2.8) is unique.

The following example illustrates our results.

Example 2.11 Consider $X=[0, \infty)$ with the usual metric. If $T: X \rightarrow X$ is the mapping defined as

$$
T(\varkappa)=\ln \left(1+5 \varkappa^{2}\right), \quad \forall \varkappa \in X,
$$

then $T$ is not a contraction mapping. Also, define $\theta: X \times X \rightarrow[0, \infty)$ as follows:

$$
\theta(\varkappa, \zeta)= \begin{cases}1, & \varkappa, \zeta \in\left[0, \frac{1}{10}\right] \\ 0, & \text { otherwise }\end{cases}
$$

Let $\vartheta(t)=t$, for all $t \geq 0$, and $h: X \times X \rightarrow[0,1)$ be a function defined by

$$
h(\varkappa, \zeta)= \begin{cases}\frac{\arctan 5\left|\varkappa^{2}-\zeta^{2}\right|}{5\left|\varkappa^{2}-\zeta^{2}\right|}, & \varkappa \neq \zeta, \\ 0, & \varkappa=\zeta .\end{cases}
$$

In the sequel, we show that $T$ is a generalized $\theta-h-\vartheta$-contraction type mapping. It is easy to see that $h \in \mathcal{A}(\mathcal{R})$ and $\vartheta \in \Phi$. Since

$$
\ln (1+t) \leq \arctan (t) \quad \text { for all } t \in[0,1]
$$

thus, for all $\varkappa, \zeta \in\left[0, \frac{1}{10}\right]$, we get

$$
\begin{aligned}
\theta(\varkappa, \zeta) \vartheta(d(T \varkappa, T \zeta)) & =\left|\ln \left(\frac{1+4 \varkappa^{2}}{1+\zeta^{2}}\right)\right| \\
& \leq \ln \left(1+5\left|\varkappa^{2}-\zeta^{2}\right|\right) \\
& \leq \arctan \left(5\left|\varkappa^{2}-\zeta^{2}\right|\right)
\end{aligned}
$$




$$
\begin{aligned}
& =\frac{\arctan \left(5\left|\varkappa^{2}-\zeta^{2}\right|\right)}{5\left|\varkappa^{2}-\zeta^{2}\right|} 5\left|\varkappa^{2}-\zeta^{2}\right| \\
& \leq \frac{\arctan \left(5\left|\varkappa^{2}-y^{2}\right|\right)}{5\left|\varkappa^{2}-\zeta^{2}\right|}|\varkappa-\zeta| \\
& =h(\varkappa, \zeta) \vartheta(d(\varkappa, \zeta)) \\
& \leq h(\varkappa, \zeta) \vartheta(R(\varkappa, \zeta)) .
\end{aligned}
$$

If $\varkappa>\frac{1}{10}$ or $\zeta>\frac{1}{10}$, then $\theta(\varkappa, \zeta)=0$, and so $\theta(\varkappa, \zeta) \vartheta(d(T \varkappa, T \zeta)) \leq h(\varkappa, \zeta) \vartheta(R(\varkappa, \zeta))$. Hence $T$ is a generalized $\theta-h-\vartheta$-contraction type mapping. Obviously, other hypotheses of Theorem 2.2 are satisfied. Therefore $T$ has a fixed point. Note that $\varkappa^{*}=0$ is the fixed point of $T$.

\section{Particular cases}

In this section, we consider some immediate consequences of our main results. We start this section by introducing the following class of auxiliary mappings:

Let $\Psi$ be the class of all upper semi-continuous from the right functions $\psi:[0, \infty) \rightarrow$ $[0, \infty)$ such that $\psi^{-1}\{0\}=\{0\}$ and $\psi(t)<t$ for all $t>0$.

First of all, we note that Theorem 2.9 (respectively, Theorem 2.2 and Theorem 2.6) is still valid if we replace the contraction inequality (2.1) with inequality (2.2) or inequality (2.3).

For this goal, we first define the following inequalities on a quadruplet $(X, d, T, \theta)$ :

$\left(I_{7}\right) \theta(\varkappa, \zeta) \vartheta(d(T \varkappa, T \zeta)) \leq \gamma(\vartheta(R(\varkappa, \zeta))) \vartheta(R(\varkappa, \zeta))$ for each $\varkappa, \zeta \in X$, where $\vartheta \in \Phi$, $\gamma \in \mathcal{G}$, and $R(\varkappa, \zeta)$ is as in $(2.1)$;

$\left(I_{8}\right) \vartheta(d(T \varkappa, T \zeta)) \leq \gamma(\vartheta(R(\varkappa, \zeta))) \vartheta(R(\varkappa, \zeta))$ for all $\varkappa, \zeta \in X$, where $\vartheta \in \Phi, \gamma \in \mathcal{G}$, and $R(\varkappa, \zeta)$ is as in $(2.1)$;

$\left(I_{9}\right) \theta(\varkappa, \zeta) d(T \varkappa, T \zeta) \leq \gamma(\vartheta(M(\varkappa, \zeta))) \vartheta(M(\varkappa, \zeta))$ for all $\varkappa, \zeta \in X$; where $\vartheta \in \Phi, \gamma \in \mathcal{G}$, and $M(\varkappa, \zeta)$ is as in (2.3) (named "generalized $\theta$-Geraghty contraction mappings");

(I10) $\theta(\varkappa, \zeta) d(T \varkappa, T \zeta) \leq \gamma((d(\varkappa, \zeta))) d(\varkappa, \zeta)$ for all $\varkappa, \zeta \in X$;

$\left(I_{11}\right) \vartheta(d(T \varkappa, T \zeta)) \leq \vartheta(d(\varkappa, \zeta))-\psi(d(\varkappa, \zeta))$ for all $\varkappa, \zeta \in X$, where $\vartheta, \psi \in \Phi$;

$\left(I_{12}\right) d(T \varkappa, T \zeta) \leq \psi(d(\varkappa, \zeta))$ for all $\varkappa, \zeta \in X$, where $\psi \in \Psi$.

Corollary 3.1 For $(X, d, T, \theta)$, we suppose that the following conditions are satisfied:

(i) inequality $\left(I_{7}\right)$ is satisfied;

(ii) $T$ is triangular $\theta$-orbital admissible and either $T$ is continuous or $X$ is $\theta$-regular;

(iii) there exists $\varkappa_{0} \in X$ such that $\theta\left(\varkappa_{0}, T \varkappa_{0}\right) \geq 1$.

Then $T$ has a fixed point.

Proof Define $h: X \times X \rightarrow[0, \infty)$ by

$$
h(\varkappa, \zeta)=\gamma(\vartheta(R(\varkappa, \zeta)) \text { for all } \varkappa, \zeta \in X
$$

Suppose that $\left\{\varkappa_{n}\right\},\left\{\zeta_{n}\right\} \subseteq X$ are such that $\lim _{n \rightarrow \infty} h\left(\varkappa_{n}, \zeta_{n}\right)=1$. Then

$$
\lim _{n \rightarrow \infty} \vartheta\left(R\left(\varkappa_{n}, \zeta_{n}\right)=0\right.
$$


Since $\vartheta$ is continuous and $\vartheta^{-1}\{0\}=0$, then $\lim _{n \rightarrow \infty} R\left(\varkappa_{n}, \zeta_{n}\right)=0$. This implies that

$$
\lim _{n \rightarrow \infty} d\left(\varkappa_{n}, \zeta_{n}\right)=\lim _{n \rightarrow \infty} d\left(\varkappa_{n}, T \varkappa_{n}\right)=\lim _{n \rightarrow \infty} d\left(\zeta_{n}, T \zeta_{n}\right)=0
$$

Hence $h \in \mathcal{A}(X)$, and so by $\left(I_{7}\right)$ we have

$$
\theta(\varkappa, \zeta) \vartheta(d(T \varkappa, T \zeta)) \leq h(\varkappa, \zeta) \vartheta(R(\varkappa, \zeta)) \quad \text { for all } \varkappa, \zeta \in X
$$

Therefore $T$ is a generalized $\theta-h-\vartheta$-contraction type mapping. Also applying (3.1) and triangular inequality implies that $\lim _{n \rightarrow \infty} d\left(T \varkappa_{n}, T \zeta_{n}\right)=0$. Hence all the hypotheses of Theorems 2.2 and 2.6 are satisfied. Thus $T$ has a fixed point $\varkappa^{*} \in X$, and $\left\{T^{n} x_{0}\right\}$ converges to $\varkappa^{*}$.

Corollary 3.2 On a setting $(X, d, T, \theta)$, if $\left(I_{8}\right)$ is fulfilled with the assumption that $T$ is continuous, then there exists a unique fixed point.

Sketch of the proof If $\theta(\varkappa, \zeta)=1$ in Corollary 3.1 for all $\varkappa, \zeta \in X$, then deduce the existence of a fixed point. Uniqueness is followed by Theorem 2.9 .

We deduce the main results of [17] as follows.

Corollary 3.3 ([17]) On a setting $(X, d, T, \theta)$, if $\left(I_{9}\right)$ is fulfilled with the following assumptions:

(a) $T$ is triangular $\theta$-orbital admissible and either $T$ is continuous or $X$ is $\theta$-regular;

(b) there exists $\varkappa_{0} \in X$ such that $\theta\left(\varkappa_{0}, T \varkappa_{0}\right) \geq 1$;

then $T$ has a fixed point.

Proof Define $h: X \times X \rightarrow[0, \infty)$ by

$$
h(\varkappa, \zeta)=\gamma(\vartheta(M(\varkappa, \zeta)) \quad \text { for all } \varkappa, \zeta \in X
$$

Similar to Corollary 3.1, we can show that $h \in \mathcal{A}(X)$ and, for any sequences $\left\{\varkappa_{n}\right\},\left\{\zeta_{n}\right\} \subseteq X$,

$$
\lim _{n \rightarrow \infty} h\left(\varkappa_{n}, \zeta_{n}\right)=1 \Longrightarrow \lim _{n \rightarrow \infty} d\left(T \varkappa_{n}, T \zeta_{n}\right)=0
$$

Since $\vartheta$ is a non-decreasing function, then for all $\varkappa, \zeta \in X$,

$$
\theta(\varkappa, \zeta) \vartheta(d(T \varkappa, T \zeta)) \leq h(\varkappa, \zeta) \vartheta(M(\varkappa, \zeta)) \leq h(\varkappa, \zeta) \vartheta(R(\varkappa, \zeta))
$$

Therefore $T$ is a generalized $\theta-h-\vartheta$-contraction mapping. Hence all the hypotheses of Theorem 2.2 and Theorem 2.6 are satisfied, which implies that $T$ has a fixed point $\varkappa^{*} \in X$, and $\left\{T^{n} x_{0}\right\}$ converges to $\varkappa^{*}$.

Define $\vartheta:[0, \infty) \rightarrow[0, \infty)$ by $\vartheta(t)=t$ for all $t \in[0, \infty)$. Similar to Corollary 3.3, we can prove that generalized $\theta$-Geraghty contraction mappings are as a subclass of generalized $\theta-h-\vartheta$-contraction type mappings. Applying Theorem 2.2 and Theorem 2.6, we have the following corollaries. 
Corollary 3.4 ([16]) On a setting $(X, d, T, \theta)$, if $\left(I_{9}\right)$ is fulfilled with the following assumptions:

(a) $T$ is triangular $\theta$-orbital admissible and either $T$ is continuous or $X$ is $\theta$-regular;

(b) there exists $\varkappa_{0} \in X$ such that $\theta\left(\varkappa_{0}, T \varkappa_{0}\right) \geq 1$;

then $T$ has a fixed point.

Corollary 3.5 (Geraghty fixed point theorem [5]) On a setting $(X, d, T, \theta)$, if $\left(I_{10}\right)$ is fulfilled, then there exists a unique fixed point.

Proof Define $\vartheta:[0, \infty) \rightarrow[0, \infty)$ and $h: X \times X \rightarrow[0, \infty)$ by

$$
h(\varkappa, \zeta)=\gamma(d(\varkappa, \zeta)), \quad \forall \varkappa, \zeta \in X \text { and } \vartheta(t)=t, \forall t \in[0, \infty)
$$

Applying Example 1.2 implies that $T$ is a continuous $\theta-h-\vartheta$-contraction mapping. Obviously the hypotheses of Corollary 3.2 are satisfied. Therefore $T$ has a fixed point $\varkappa^{*} \in X$, and $\left\{T^{n} x_{0}\right\}$ converges to $\varkappa^{*}$.

Corollary 3.6 ( $\vartheta-\psi$-weakly contractive fixed point theorem [12]) On a setting $(X, d, T, \theta)$, if $\left(I_{11}\right)$ is fulfilled with the assumption that $T$ is continuous, then $T$ has a unique fixed point.

Proof Define $h: X \times X \rightarrow[0,1)$ by

$$
h(\varkappa, \zeta)= \begin{cases}\frac{\vartheta(d(\varkappa, \zeta))-\psi(d(\varkappa, \zeta))}{\vartheta(d(\varkappa, \zeta))} & \text { if } \varkappa \neq \zeta \\ 0 & \text { if } \varkappa=\zeta .\end{cases}
$$

Let $\left\{\varkappa_{n}\right\},\left\{\zeta_{n}\right\} \subseteq X$ be such that the sequence $\left\{d\left(\varkappa_{n}, \zeta_{n}\right)\right\}$ is decreasing and $\lim _{n \rightarrow \infty} d\left(\varkappa_{n}\right.$, $\left.\zeta_{n}\right)=r$. Suppose that $\lim _{n \rightarrow \infty} h\left(\varkappa_{n}, \zeta_{n}\right)=1$. We show that $\lim _{n \rightarrow \infty} d\left(\varkappa_{n}, \zeta_{n}\right)=0$. In the contrary case, let $\lim _{n \rightarrow \infty} d\left(\varkappa_{n}, \zeta_{n}\right)=r>0$. Since $\vartheta$ and $\psi$ are continuous, thus

$$
\lim _{n \rightarrow \infty} h\left(\varkappa_{n}, \zeta_{n}\right)=\lim _{n \rightarrow \infty} \frac{\vartheta\left(d\left(\varkappa_{n}, \zeta_{n}\right)\right)-\psi\left(d\left(\varkappa_{n}, \zeta_{n}\right)\right)}{\vartheta\left(d\left(\varkappa_{n}, \zeta_{n}\right)\right)}=\frac{\vartheta(r)-\psi(r)}{\vartheta(r)}=1,
$$

which implies that $\psi(r)=0$, and so $r=0$. This is a contradiction. Eventually, we have

$$
\lim _{n \rightarrow \infty} d\left(\varkappa_{n}, \zeta_{n}\right)=0,
$$

which yields $h \in \mathcal{A}(X)$. Applying $\left(I_{11}\right)$ and (3.2), we conclude that, for all $\varkappa, \zeta \in X$,

$$
\vartheta(d(T \varkappa, T \zeta)) \leq h(\varkappa, \zeta) \vartheta(d(\varkappa, \zeta)) \leq h(\varkappa, \zeta) \vartheta(R(\varkappa, \zeta)) .
$$

Hypotheses of Corollary 3.2 are satisfied. Hence the mapping $T$ has a unique fixed point.

Corollary 3.7 (Boyd and Wong fixed point theorem [6]) On a setting $(X, d, T, \theta)$, if $\left(I_{12}\right)$ is fulfilled with the assumption that $T$ is continuous, then there exists a unique fixed point.

Proof Consider $h: X \times X \rightarrow[0,1)$ by

$$
h(\varkappa, \zeta)= \begin{cases}\frac{\psi(d(\varkappa, \zeta))}{d(\varkappa, \zeta)} & \text { if } \varkappa \neq \zeta \\ 0 & \text { if } \varkappa=\zeta .\end{cases}
$$


Let $\left\{\varkappa_{n}\right\}$ and $\left\{\zeta_{n}\right\}$ be sequences in $X$ such that the sequence $\left\{d\left(\varkappa_{n}, \zeta_{n}\right)\right\}$ is decreasing and convergent. Suppose that $\lim _{n \rightarrow \infty} h\left(\varkappa_{n}, \zeta_{n}\right)=1$, we prove that $\lim _{n \rightarrow \infty} d\left(\varkappa_{n}, \zeta_{n}\right)=0$. In the contrary case, let $\lim _{n \rightarrow \infty} d\left(\varkappa_{n}, \zeta_{n}\right)=r>0$. Since $\psi$ is upper semi-continuous from the right, thus

$$
1=\lim _{n \rightarrow \infty} h\left(\varkappa_{n}, \zeta_{n}\right)=\lim _{n \rightarrow \infty} \frac{\psi\left(d\left(\varkappa_{n}, \zeta_{n}\right)\right)}{d\left(\varkappa_{n}, \zeta_{n}\right)} \leq \frac{\psi(r)}{r}
$$

which yields $\psi(r) \geq r$, a contradiction. Therefore

$$
\lim _{n \rightarrow \infty} d\left(\varkappa_{n}, \zeta_{n}\right)=0
$$

This implies that $h \in \mathcal{A}(X)$. Let $\vartheta(t)=t$ for all $t \in[0, \infty)$. From $\left(I_{12}\right)$ we conclude that

$$
\vartheta(d(T \varkappa, T \zeta)) \leq h(\varkappa, \zeta) \vartheta(d(\varkappa, \zeta)), \quad \forall \varkappa, \zeta \in X
$$

Hypotheses of Corollary 3.2 are satisfied. Therefore the mapping $T$ has a unique fixed point.

Remark 3.8 We underline that our main results are Theorem 2.9 (which contains Theorem 2.2, Theorem 2.6) and Theorem 2.10 (which contains Theorem 2.4, Theorem 2.8). Here, we consider only a few of immediate consequences of Theorem 2.9 but not Theorem 2.10. It is clear that from both Theorem 2.10 and Theorem 2.9 we derive and list more consecutive results.

\section{Application to nonlinear differential equations in the frame of fractional derivatives with singular kernels}

In this section we discuss the application of our results to the existence of solutions for Caputo fractional boundary value problem of order $\beta \in(1,2]$ with integral boundary condition type. As the definition of the Caputo fractional derivative is given below, it is obvious that the kernel has singularity.

Let $\beta$ be a positive real number and $\Gamma$ be a gamma function. For a continuous function $g:[0, \infty) \rightarrow \mathbb{R}$, the Caputo derivative of fractional order $\beta$ is defined as

$$
\left({ }_{0}^{C} D^{\beta} g\right)(t)=\frac{1}{\Gamma(n-\beta)} \int_{0}^{t}(t-s)^{n-\beta-1} g^{(n)}(s) d s, \quad n=[\beta]+1 .
$$

Consider the following nonlinear fractional differential equation:

$$
\left({ }_{0}^{C} D^{\beta} u\right)(t)=f(t, u(t)), \quad t \in I \text { and } 1<\beta \leq 2,
$$

via the integral boundary condition

$$
u(0)=0, u(1)=\int_{0}^{r} u(s) d s, \quad r \in(0,1)
$$


where $u \in C[0,1]$ and $f: I \times \mathbb{R} \rightarrow \mathbb{R}$ is a continuous function. We define the operator equation $T: C[0,1] \rightarrow C[0,1]$ as follows:

$$
\begin{aligned}
T(u)(t)= & \frac{1}{\Gamma(\beta)} \int_{0}^{t}(t-s)^{\beta-1} f(s, u(s)) d s \\
& -\frac{2 t}{\left(2-r^{2}\right) \Gamma(\beta)} \int_{0}^{1}(1-s)^{\beta-1} f(s, u(s)) d s \\
& +\frac{2 t}{\left(2-r^{2}\right) \Gamma(\beta)} \int_{0}^{r}\left(\int_{0}^{s}(s-z)^{\beta-1} f(z, u(z)) d z\right) d s, \quad t \in I .
\end{aligned}
$$

We know that $u \in C[0,1]$ is a solution of $(2.18)$ if and only if $u \in C[0,1]$ is the fixed point of the mapping $T$. Suppose the following conditions:

$\left(\mathrm{H}_{1}\right)$ There exist $\xi: \mathbb{R}^{2} \rightarrow \mathbb{R}$ and $\psi \in \Psi$ such that, for all $t \in I$ and $a, b \in \mathbb{R}$ with $\xi(a, b) \geq$ 0 ,

$$
|f(t, a)-f(t, b)| \leq K_{1} \psi(|a-b|), \quad K_{1}=\frac{\Gamma(\beta+2)}{5+3 \beta} ;
$$

$\left(\mathrm{H}_{2}\right)$ There exists $u_{0} \in C[0,1]$ such that $\xi\left(u_{0}(t), T\left(u_{0}(t)\right)\right) \geq 0$ for all $t \in I$;

$\left(\mathrm{H}_{3}\right)$ For all $t \in I$ and $u, v \in C[0,1]$,

$$
\xi(u(t), v(t)) \geq 0 \quad \text { implies that } \quad \xi(T(u(t)), T(v(t))) \geq 0 \text {; }
$$

$\left(\mathrm{H}_{4}\right)$ Let $\left\{u_{n}\right\}$ be a sequence in $C[0,1]$ such that $u_{n} \rightarrow u$ in $C[0,1]$. Let, for all $t \in I$,

$$
\xi\left(u_{n}(t), u_{n+1}(t)\right) \geq 0, \quad \forall n \in \mathbb{N} \quad \Longrightarrow \quad \xi\left(u_{n}(t), u(t)\right) \geq 0 .
$$

Theorem 4.1 Let conditions $\left(\mathrm{H}_{1}\right)-\left(\mathrm{H}_{4}\right)$ be satisfied. Then $T$ has at least one fixed point $u^{*} \in C[0,1]$.

Proof We prove that $T$ is a generalized $\alpha-h-\vartheta$-contraction mapping. Now, let $u, v \in C[0,1]$ such that, for all $t \in I, \xi(u(t), v(t)) \geq 0$. Applying $\left(\mathrm{H}_{1}\right)$,

$$
\begin{aligned}
|T(u)(t)-T(v)(t)|= & \mid \frac{1}{\Gamma(\beta)} \int_{0}^{t}(t-s)^{\beta-1} f(s, u(s)) d s \\
& -\frac{2 t}{\left(2-r^{2}\right) \Gamma(\beta)} \int_{0}^{1}(1-s)^{\beta-1} f(s, u(s)) d s \\
& +\frac{2 t}{\left(2-r^{2}\right) \Gamma(\beta)} \int_{0}^{r}\left(\int_{0}^{s}(s-z)^{\beta-1} f(z, u(z)) d z\right) d s \\
& -\frac{1}{\Gamma(\beta)} \int_{0}^{t}(t-s)^{\beta-1} f(s, v(s)) d s \\
& +\frac{2 t}{\left(2-r^{2}\right) \Gamma(\beta)} \int_{0}^{1}(1-s)^{\beta-1} f(s, v(s)) d s \\
& -\frac{2 t}{\left(2-r^{2}\right) \Gamma(\beta)} \int_{0}^{r}\left(\int_{0}^{s}(s-z)^{\beta-1} f(z, v(z)) d z\right) d s \mid \\
\leq & \frac{1}{\Gamma(\beta)} \int_{0}^{t}|t-s|^{\beta-1}|f(s, u(s))-f(s, v(s))| d s
\end{aligned}
$$




$$
\begin{aligned}
& +\frac{2 t}{\left(2-r^{2}\right) \Gamma(\beta)} \int_{0}^{1}(1-s)^{\beta-1}|f(s, u(s))-f(s, v(s))| d s \\
& +\frac{2 t}{\left(2-r^{2}\right) \Gamma(\beta)} \int_{0}^{r}\left|\int_{0}^{s}(s-z)^{\beta-1}\right| f(z, u(z))-f(z, v(z))|d z| d s \\
\leq & \frac{1}{\Gamma(\beta)} \int_{0}^{t}|t-s|^{\beta-1} K_{1} \psi(|v(s)-u(s)|) d s \\
& +\frac{2 t}{\left(2-r^{2}\right) \Gamma(\beta)} \int_{0}^{1}|1-s|^{\beta-1} K_{1} \psi(|v(s)-u(s)|) d s \\
& +\frac{2 t}{\left(2-r^{2}\right) \Gamma(\beta)} \int_{0}^{r}\left(\int_{0}^{s}|s-z|^{\beta-1} K_{1} \psi(|v(z)-u(z)|) d z\right) d s \\
\leq & K_{1} \psi\left(\|v-u\|_{\infty}\right) \times \sup _{t \in(0,1)}\left(\frac{1}{\Gamma(\beta)} \int_{0}^{t}|t-s|^{\beta-1} d s\right. \\
& +\frac{2 t}{\left(2-r^{2}\right) \Gamma(\beta)} \int_{0}^{1}|1-s|^{\beta-1} d s \\
& \left.+\frac{2 t}{\left(2-r^{2}\right) \Gamma(\beta)} \int_{0}^{r} \int_{0}^{s}|s-z|^{\beta-1} d z d s\right) \\
\leq & \psi\left(\|v-u\|_{\infty}\right)=\psi(d(u, v)) .
\end{aligned}
$$

We define $\alpha: C[0,1] \times C[0,1] \rightarrow[0, \infty)$ by

$$
\alpha(u, v)= \begin{cases}1, & \xi(u(t), v(t)) \geq 0 \forall t \in I \\ 0, & \text { otherwise }\end{cases}
$$

and

$$
h(u, v)= \begin{cases}\frac{\psi(d(u, v))}{d(u, v)} & \text { if } u \neq v \\ 0 & \text { if } u=v .\end{cases}
$$

Then, for all $u, v \in C[0,1]$, we have

$$
\begin{aligned}
\alpha(u, v) d(T u, T v) & \leq \psi(d(u, v))=\frac{\psi(d(u, v))}{d(u, v)} d(u, v) \\
& =h(u, v) d(u, v) \\
& \leq h(u, v) R(u, v) .
\end{aligned}
$$

Let $\vartheta(x)=x$ for all $x \in[0, \infty)$. Then $T$ is a generalized $\alpha-h-\vartheta$-contraction type mapping. One can prove that all the hypotheses of Theorem 2.6 are satisfied. Therefore there exists $u^{*} \in C[0,1]$ such that $T u^{*}=u^{*}$.

\section{Application to equations in the frame of a Caputo type fractional derivatives}

Consider the following nonlinear fractional differential equation:

$$
\left({ }_{0}^{C} D^{\beta, \rho} u(t)\right)=f(t, u(t)), \quad t \in I \text { and } 1<\beta \leq 2, \rho \in(0,1]
$$


with the exponentially weighted integral boundary condition

$$
u(0)=0, u(1)=\int_{0}^{r} e^{\lambda s} u(s) d s, \quad r \in(0,1), \lambda=\frac{1-\rho}{\rho},
$$

where $u \in C[0,1]$ and $f: I \times \mathbb{R} \rightarrow \mathbb{R}$ is a continuous function. The fractional derivative in (5.1) is the fractional derivative that was found together with its discrete version in [2] and [3] in an attempt to find the fractional operators generated by the local proportional derivative proposed in [36] as a modified version of the conformable derivative [37, 38]. It turned out that this derivative is so interesting in the sense that it is a constant multiple of the tempered derivative discussed in [39-41].

Apply the fractional integral ${ }_{0} I^{\beta, \rho}$ to (5.1) and make use of Theorem 5.2 with $n=2$ in [2] to reach the solution integral representation

$$
u(t)=c_{0} e^{-\lambda t}+c_{1} t e^{-\lambda t}+\frac{1}{\rho^{\beta} \Gamma(\beta)} \int_{0}^{t} e^{-\lambda(t-s)}(t-s)^{\beta-1} f(s, u(s)) d s .
$$

The boundary condition $u(0)=0$ implies that $c_{0}=0$. If we integrate the solution $u(t)$ in (5.3) with $c_{0}=0$ from 0 to $r$, then we have

$$
\int_{0}^{r} u(s) e^{\lambda s} d s=c_{1} \int_{0}^{s} s d s+\frac{1}{\rho^{\beta} \Gamma(\beta)} \int_{0}^{r} \int_{0}^{s} e^{\lambda z}(s-z)^{\beta-1} f(z, u(z)) d z d s .
$$

Now, if we use the integral boundary condition, then we conclude that

$$
\begin{aligned}
c_{1}= & \frac{2}{\rho^{\beta} \Gamma(\beta)\left[2 e^{-\lambda}-r^{2}\right]}\left[\int_{0}^{r} \int_{0}^{s} e^{\lambda z}(s-z)^{\beta-1} f(z, u(z)) d z d s\right. \\
& \left.-\int_{0}^{1} e^{-\lambda(1-s)}(1-s)^{\beta-1} f(s, u(s)) d s\right] .
\end{aligned}
$$

Hence, we obtain the solution will take the form

$$
\begin{aligned}
u(t)= & \frac{2 t e^{-\lambda t}}{\rho^{\beta} \Gamma(\beta)\left[e^{-\lambda}-r^{2}\right]}\left[\int_{0}^{r} \int_{0}^{s} e^{\lambda z}(s-z)^{\beta-1} f(z, u(z)) d z d s\right. \\
& \left.-\int_{0}^{1} e^{-\lambda(1-s)}(1-s)^{\beta-1} f(s, u(s)) d s\right] \\
& +\frac{1}{\rho^{\beta} \Gamma(\beta)} \int_{0}^{t} e^{-\lambda(t-s)}(t-s)^{\beta-1} f(s, u(s)) d s .
\end{aligned}
$$

We define the operator equation $T^{\rho}: C[0,1] \rightarrow C[0,1]$ as follows:

$$
\begin{aligned}
\left(T^{\rho} u\right)(t)= & \frac{2 t e^{-\lambda t}}{\rho^{\beta} \Gamma(\beta)\left[e^{-\lambda}-r^{2}\right]}\left[\int_{0}^{r} \int_{0}^{s} e^{\lambda z}(s-z)^{\beta-1} f(z, u(z)) d z d s\right. \\
& \left.-\int_{0}^{1} e^{-\lambda(1-s)}(1-s)^{\beta-1} f(s, u(s)) d s\right] \\
& +\frac{1}{\rho^{\beta} \Gamma(\beta)} \int_{0}^{t} e^{-\lambda(t-s)}(t-s)^{\beta-1} f(s, u(s)) d s .
\end{aligned}
$$

It is now straightforward that $u \in C[0,1]$ is a solution of (5.1)-(5.2) if and only if $u \in C[0,1]$ is the fixed point of the mapping $T^{\rho}$. Suppose the following conditions: 
$\left(p \mathrm{H}_{1}\right)$ There exist $\xi: \mathbb{R}^{2} \rightarrow \mathbb{R}$ and $\psi \in \Psi$ such that, for all $t \in I$ and $a, b \in \mathbb{R}$ with $\xi(a, b) \geq$ 0

$$
|f(t, a)-f(t, b)| \leq K_{\rho} \psi(|a-b|)
$$

where $K_{\rho}=\left[\frac{E_{1, \beta+1}(\lambda, 1)}{\rho^{\beta}}+\frac{2 C(\rho) e^{-\lambda} E_{1, \beta+1}(\lambda, 1)}{\left(2 e^{-\lambda}-r^{2}\right) \rho^{\beta}}+\frac{2 C(\rho) E_{1, \beta+2}(\lambda, 1)}{\left(2 e^{-\lambda}-r^{2}\right) \rho^{\beta}}\right]^{-1}$.

We recall the modified version of the Mittag-Leffler (ML) function by (see, for example, [20] for ML function and [42] for the modified ML function)

$$
\begin{aligned}
E_{\alpha, \beta}(\lambda, z) & =z^{\beta-1} E_{\alpha, \beta}\left(\lambda z^{\alpha}\right) \\
& =\sum_{k=0}^{\infty} \lambda^{k} \frac{z^{\alpha k+\beta-1}}{\Gamma(\alpha k+\beta)} \quad(0 \neq \lambda \in \mathbb{R}, z, \beta \in \mathbb{C} ; \operatorname{Re}(\alpha)>0)
\end{aligned}
$$

$\left(p \mathrm{H}_{2}\right)$ There exists $u_{0} \in C[0,1]$ such that $\xi\left(u_{0}(t), T^{\rho}\left(u_{0}(t)\right)\right) \geq 0$ for all $t \in I$;

$\left(p \mathrm{H}_{3}\right)$ For all $t \in I$ and $u, v \in C[0,1]$,

$$
\xi(u(t), v(t)) \geq 0 \quad \text { implies that } \quad \xi\left(T^{\rho}(u(t)), T^{\rho}(v(t))\right) \geq 0
$$

$\left(p \mathrm{H}_{4}\right)$ Let $\left\{u_{n}\right\}$ be a sequence in $C[0,1]$ such that $u_{n} \rightarrow u$ in $C[0,1]$. Let, for all $t \in I$,

$$
\xi\left(u_{n}(t), u_{n+1}(t)\right) \geq 0, \quad \forall n \in \mathbb{N} \quad \Longrightarrow \quad \xi\left(u_{n}(t), u(t)\right) \geq 0
$$

Before proving our existence theorem, we state and prove the following lemma.

Lemma 5.1 Assume $\lambda=\frac{1-\rho}{\rho}$ and $C(\rho)=\left\{\begin{array}{ll}\frac{1}{\lambda e}, & 0<\rho \leq \frac{1}{2}, \\ e^{-\lambda}, & \frac{1}{2}<\rho \leq 1 .\end{array}\right.$ Then the function $f(t)=t e^{-\lambda t}$ satisfies $f(t) \leq C(\rho)$ for all $t \in[0,1]$.

Proof The critical value $t^{*}=\frac{1}{\lambda}$ of the function $f(t)$ lies in the interval $I$ only if $0<\rho \leq \frac{1}{2}$ and hence the maximum of $f(t)$ is equal to $f\left(t^{*}\right)=\frac{1}{\lambda e}$. On the other hand, the critical value of $f(t)$ lies outside $I$ if $\frac{1}{2}<\rho \leq 1$ and in this case $f(t)$ is increasing in $I$, and hence the maximum of $f$ is equal to $f(1)=e^{-\lambda}$.

Theorem 5.2 Let conditions $\left(p \mathrm{H}_{1}\right)-\left(p \mathrm{H}_{4}\right)$ be satisfied. Then $T^{\rho}$ has at least one solution $u^{*} \in C[0,1]$.

Proof We prove that $T^{\rho}$ is a generalized $\alpha-h-\vartheta$-contraction mapping. Now, let $u, v \in$ $C[0,1]$ such that, for all $t \in I, \xi(u(t), v(t)) \geq 0$. Applying $\left(p \mathrm{H}_{1}\right)$, then the proof is as in Theorem 4.1, except that we have

$$
\begin{aligned}
& \left|T^{\rho}(u)(t)-T^{\rho}(v)(t)\right| \\
& \leq \frac{1}{\rho^{\beta} \Gamma(\beta)} \int_{0}^{t} e^{-\lambda(t-s)}|t-s|^{\beta-1} K_{\rho} \psi(|v(s)-u(s)|) d s \\
& \quad+\frac{2 t e^{-\lambda t}}{\left(2 e^{-\lambda}-r^{2}\right) \rho^{\beta} \Gamma(\beta)} \int_{0}^{1} e^{-\lambda(1-s)}|1-s|^{\beta-1} K_{\rho} \psi(|v(s)-u(s)|) d s \\
& \quad+\frac{2 t e^{-\lambda t}}{\left(2 e^{-\lambda}-r^{2}\right) \rho^{\beta} \Gamma(\beta)} \int_{0}^{r}\left(\int_{0}^{s} e^{\lambda z}|s-z|^{\beta-1} K_{\rho} \psi(|v(z)-u(z)|) d z\right) d s
\end{aligned}
$$




$$
\begin{aligned}
\leq & K_{\rho} \psi\left(\|v-u\|_{\infty}\right) \times \sup _{t \in(0,1)}\left(\frac{1}{\rho^{\beta} \Gamma(\beta)} \int_{0}^{t} e^{-\lambda(t-s)}|t-s|^{\beta-1} d s\right. \\
& +\frac{2 t e^{-\lambda t}}{\left(2 e^{-\lambda}-r^{2}\right) \Gamma(\beta) \rho^{\beta}} \int_{0}^{1} e^{-\lambda(1-s)}|1-s|^{\beta-1} d s \\
& \left.+\frac{2 t e^{-\lambda t}}{\left(2 e^{-\lambda}-r^{2}\right) \rho^{\beta} \Gamma(\beta)} \int_{0}^{r} \int_{0}^{s} e^{\lambda z}|s-z|^{\beta-1} d z d s\right) \\
\leq & K_{\rho} \psi\left(\|v-u\|_{\infty}\right)\left[\frac{E_{1, \beta+1}(\lambda, 1)}{\rho^{\beta}}+\frac{2 C(\rho) e^{-\lambda} E_{1, \beta+1}(\lambda, 1)}{\left(2 e^{-\lambda}-r^{2}\right) \rho^{\beta}}+\frac{2 C(\rho) E_{1, \beta+2}(\lambda, 1)}{\left(2 e^{-\lambda}-r^{2}\right) \rho^{\beta}}\right] \\
\leq & \psi\left(\|v-u\|_{\infty}\right)=\psi(d(u, v)) .
\end{aligned}
$$

In the proof we have used the fact that ${ }_{0} I^{\beta} E_{\alpha, \mu}(\lambda, t)=E_{\alpha, \beta+\mu}(\lambda, t)$ and $e^{\lambda t}=E_{1,1}(\lambda, t)$, where

$$
{ }_{0} I^{\beta} f(t)=\frac{1}{\Gamma(\beta)} \int_{0}^{t}(t-s)^{\beta-1} f(s) d s
$$

is the left Riemann-Liouville fractional integral of order $\beta$ with $\operatorname{Re}(\beta)>0$.

Remark 5.3 Notice that, if $\rho=1$, then $\lambda=0$, and hence $C(1)=1$ and $E_{1, \beta+1}(0,1)=\frac{1}{\Gamma(\beta+1)}$. Therefore, Theorem 4.1 is the $\rho=1$ version of Theorem 5.2. In fact, from the proof Theorem 4.1 or Theorem 5.2, the constant $K_{1}$ in Theorem 4.1 can be chosen sharper and depending on $r$ by

$$
K_{1}=\frac{\left(2-r^{2}\right) \Gamma(\beta+2)}{\left(4-r^{2}\right) \beta+6-r^{2}} .
$$

Remark 5.4 If we use in Theorem 5.2 a non-weighted integral boundary condition as in Theorem 4.1, then we must assume that $0<\rho<1$. Since the case $\rho=1$ implies that $\lambda=0$, the evaluation of $\int_{0}^{r} s e^{-\lambda s} d s=\int_{0}^{r} s d s$ by parts will lead to error. After similar calculations to those in Theorem 5.2, the solution will have the form

$$
\begin{aligned}
u(t)= & \frac{t e^{-\lambda t} T(\lambda, r)}{\rho^{\beta} \Gamma(\beta)}\left[\int_{0}^{r} \int_{0}^{s} e^{-\lambda(s-z)}(s-z)^{\beta-1} f\left(z, u(z) d z d s-\int_{0}^{1} e^{-\lambda(1-s)} f(s, u(s)) d s\right]\right. \\
& +\frac{1}{\rho^{\beta} \Gamma(\beta)} \int_{0}^{t} e^{-\lambda(t-s)}(t-s)^{\beta-1} f(s, u(s)) d s .
\end{aligned}
$$

Then we request the constant $K_{\rho}$ to have the more complicated form

$$
\begin{aligned}
K_{\rho}= & {\left[\frac{C(\rho) T(\lambda, r) \int_{0}^{r} e^{-\lambda s} E_{1, \beta+1}(\lambda, s) d s}{\rho^{\beta}}\right.} \\
& \left.+\frac{C(\rho) T(\lambda, r) E_{1, \beta+1}(\lambda, 1) e^{\lambda}}{\rho^{\beta}}+\frac{E_{1, \beta+1}(\lambda, 1)}{\rho^{\beta}}\right]^{-1},
\end{aligned}
$$

where

$$
C(\lambda, r)=\frac{1-e^{-\lambda r}(\lambda r+1)}{\lambda^{2}}, \quad T(\lambda, r)=\frac{1}{e^{-\lambda}-C(\lambda, r)} .
$$




\section{Application to ordinary differential equations}

Let $X=C[0,1]$ be the space of all continuous functions defined on $I$, where $I=[0,1]$ and $u \in X$. Consider the following two-point boundary value problem of a second order differential equation:

$$
\left\{\begin{array}{l}
-u^{\prime \prime}(t)-f(t, u(t))=0 ; \quad t \in[0,1] \\
u(0)=u(1)=0
\end{array}\right.
$$

where $f:[0,1] \times \mathbb{R} \rightarrow \mathbb{R}$ is a continuous function. It is known that $u=u(t) \in C[0,1]$ is a solution of 6.1 if and only if $u \in C[0,1]$ is a solution of the integral equation

$$
u(t)=\int_{0}^{1} k(t, s) f(s, u(s)) d s
$$

where $k(t, s)$ is defined as follows:

$$
k(t, s)= \begin{cases}t(1-s), & 0 \leq t \leq s \leq 1 \\ s(1-t), & 0 \leq s \leq t \leq 1\end{cases}
$$

Theorem 6.1 Let $\psi \in \Psi$ and the following conditions be satisfied:

$\left(\mathrm{H}_{1}\right)$ There exists a function $\xi: \mathbb{R}^{2} \rightarrow \mathbb{R}$ such that, for all $t \in I$ and for all $a, b \in \mathbb{R}$ with $\xi(a, b) \geq 0$, we have

$$
|f(t, a)-f(t, b)| \leq 8 \psi(|a-b|)
$$

$\left(\mathrm{H}_{2}\right)$ There exists $u_{1} \in C[0,1]$ such that, for all $t \in I$,

$$
\xi\left(u_{1}(t), \int_{0}^{1} k(t, s) f\left(s, u_{1}(s)\right) d s\right) \geq 0
$$

$\left(\mathrm{H}_{3}\right)$ For all $t \in I$ and $u, v \in C[0,1]$,

$$
\begin{aligned}
& \xi(u(t), v(t)) \geq 0 \quad \text { implies } \\
& \xi\left(\int_{0}^{1} k(t, s) f(s, u(s)) d s, \int_{0}^{1} k(t, s) f(s, v(s)) d s\right) \geq 0
\end{aligned}
$$

$\left(\mathrm{H}_{4}\right)$ Let $\left\{u_{n}\right\}$ be a sequence in $C[0,1]$ such that $u_{n} \rightarrow u$ in $C[0,1]$. Let, for all $t \in I$ and $n \in \mathbb{N}$,

$$
\xi\left(u_{n}(t), u_{n+1}(t)\right) \geq 0 \quad \text { imply that } \quad \xi\left(u_{n}(t), u(t)\right) \geq 0 .
$$

Then boundary value problem (6.1) has a solution.

Proof We define $T: C[0,1] \rightarrow C[0,1]$ by

$$
T(u(t))=\int_{0}^{1} k(t, s) f(s, u(s)) d s, \quad \forall t \in I .
$$


Thus, a solution of problem (6.1) corresponds to a fixed point of $T$. Now our purpose is to prove that integral operator $T$ is a generalized $\alpha-h-\vartheta$-contraction.

Let $u, v \in C[0,1]$ such that $\xi(u(t), v(t)) \geq 0$ for all $t \in I$. Applying $\left(\mathrm{H}_{1}\right)$,

$$
\begin{aligned}
|T u(t)-T v(t)| & =\left|\int_{0}^{1} k(t, s)(f(s, u(s))-f(s, y(s))) d s\right| \\
& \leq \int_{0}^{1} k(t, s)|f(s, u(s))-f(s, v(s))| d s \\
& \leq \int_{0}^{1} k(t, s)(8 \psi(|u(s)-v(s)|)) d s \\
& \leq 8 \sup _{t \in I} \int_{0}^{1} k(t, s) d s \psi(d(u, v)) \\
& =\psi(d(u, v)) .
\end{aligned}
$$

We define $\alpha: C[0,1] \times C[0,1] \rightarrow[0, \infty)$ by

$$
\alpha(u, v)= \begin{cases}1 & \text { if } \xi(u(t), v(t)) \geq 0 \text { for all } t \in I \\ 0 & \text { otherwise }\end{cases}
$$

and

$$
h(u, v)= \begin{cases}\frac{\psi(d(u, v))}{d(u, v)} & \text { if } u \neq v \\ 0 & \text { if } u=v\end{cases}
$$

Then, for all $u, v \in C[0,1]$, we have

$$
\begin{aligned}
\alpha(u, v) d(T u, T v) & \leq \psi(d(u, v))=\frac{\psi(d(u, v))}{d(u, v)} d(u, v) \\
& =h(u, v) d(u, v) \\
& \leq h(u, v) R(u, v) .
\end{aligned}
$$

Let $\vartheta(x)=x$ for all $x \in[0, \infty)$. Then $T$ is a generalized $\alpha-h-\vartheta$-contraction type mapping.

Let $\left\{u_{n}\right\},\left\{v_{n}\right\}$ be sequences in $C[0,1]$ such that $\lim _{n \rightarrow \infty} h\left(u_{n}, v_{n}\right)=1$ and, for all $n \in \mathbb{N}$, $\alpha\left(u_{n}, v_{n}\right) \neq 0$. By the definition of $\alpha$, for all $n \in \mathbb{N}$ and $t \in[0,1]$, we have $\xi\left(u_{n}(t), v_{n}(t)\right) \geq 0$, and so $d\left(T u_{n}(t), T v_{n}(t)\right) \leq \psi\left(d\left(u_{n}, v_{n}\right)\right)$, which implies that, for all $n \in \mathbb{N}, d\left(T u_{n}, T v_{n}\right) \leq$ $\psi\left(d\left(u_{n}, v_{n}\right)\right)$. Since $\lim _{n \rightarrow \infty} d\left(u_{n}, v_{n}\right)=0$, therefore $\lim _{n \rightarrow \infty} d\left(T u_{n}, T v_{n}\right)=0$. This implies that condition (i) of Theorem 2.6 is satisfied. Applying conditions $\left(\mathrm{H}_{2}\right)-\left(\mathrm{H}_{4}\right)$, all the hypotheses of Theorem 2.6 are satisfied. Therefore there exists $u^{*} \in C[0,1]$ such that $T u^{*}=u^{*}$.

\section{Conclusions}

- We have extended and unified several existing results in the literature.

- We have applied our fixed point results to prove the existence of solutions for second order ordinary boundary value problems. 
- We have applied our fixed point results to the existence of solutions to fractional operators with singular kernels (the Caputo and more generally a Caputo type fractional). In the Caputo fractional boundary value problem we used integral type boundary condition, and in the Caputo type fractional boundary value problem we used a weighted integral type boundary condition. In future works, we shall apply the fixed point techniques on the fractional operators with nonsingular kernels (see, for example, $[1,23,43]$ and the references therein).

\section{Acknowledgements}

The authors thank anonymous referees for their remarkable comments, suggestion, and ideas that helped to improve this paper.

\section{Funding}

The second author (T. Abdeljawad) would like to thank Prince Sultan University for funding this work through research group Nonlinear Analysis Methods in Applied Mathematics (NAMAM), group number RG-DES-2017-01-17.

\section{Competing interests}

The authors declare that they have no competing interests.

\section{Authors' contributions}

All authors equally contributed to this manuscript and approved the final version. All authors read and approved the final version of this paper.

\section{Author details}

${ }^{1}$ Department of Medical Research, China Medical University, Taichung, Taiwan. ${ }^{2}$ Department of Mathematics and General Sciences, Prince Sultan University, Riyadh, Saudi Arabia. ${ }^{3}$ Department of Mathematics, Çankaya University, Ankara, Turkey.

\section{Publisher's Note}

Springer Nature remains neutral with regard to jurisdictional claims in published maps and institutional affiliations.

Received: 26 February 2019 Accepted: 25 September 2019 Published online: 03 October 2019

\section{References}

1. Jarad, F., Abdeljawad, T., Hammouch, Z:: On a class of ordinary differential equations in the frame of Atangana-Baleanu fractional derivative. Chaos Solitons Fractals 117, 16-20 (2018)

2. Jarad, F., Abdeljawad, T., Alzabut, J.: Generalized fractional derivatives generated by a class of local proportional derivatives. Eur. Phys. J. Spec. Top. 226, 3457-3471 (2017)

3. Abdeljawad, T., Jarad, F., Alzabut, J.: Fractional proportional differences with memory. Eur. Phys. J. Spec. Top. 226, 3333-3354 (2017)

4. Banach, S.: Sur les opérations dans les ensembles abstraits et leur application aux équations intégrales. Fundam Math. 3, 133-181 (1922)

5. Geraghty, M.: On contractive mappings. Proc. Am. Math. Soc. 40, 604-608 (1973)

6. Boyd, D.W., Wong, J.S.W.: On nonlinear contractions. Proc. Am. Math. Soc. 20, 458-464 (1969)

7. Jaggi, D.S.: Some unique fixed point theorems. Indian J. Pure Appl. Math. 8, 223-230 (1977)

8. Rhoades, B.E.: Some theorems on weakly contractive maps. Nonlinear Anal. 47(4), 2683-2693 (2001)

9. Dass, B.K., Gupta, S.: An extension of Banach contraction principle through rational expressions. Indian J. Pure Appl. Math. 6, 1455-1458 (1975)

10. Alqahtani, B., Hamzehnejadi, J., Karapınar, E., Lashkaripour, R.: Best proximity point for certain proximal contraction type mappings. J. Math. Anal. 9(5), 1-15 (2018)

11. Hamzehnejadi, J., Lashkaripour, R.: Best proximity points for generalized $\alpha$ - $\phi$-Geraghty proximal contraction mappings. Fixed Point Theory Appl. 2016, 72 (2016)

12. Dutta, P.N., Choudhury, B.S.: A generalization of contraction principle in metric spaces. Fixed Point Theory Appl., 2008, Article ID 406368 (2008)

13. Popescu, O.: Some new fixed point theorems for $\alpha$-Geraghty contractive type maps in metric spaces. Fixed Point Theory Appl. 2014, 190 (2014)

14. Samet, B., Vetro, C., Vetro, P.: Fixed point theorems for $\alpha$ - $\psi$-contractive type mappings. Nonlinear Anal. 75 , 2154-2165 (2012)

15. Karapınar, E., Samet, B.: A note on $\psi$-Geraghty type contractions. Fixed Point Theory Appl. 2014, 26 (2013)

16. Cho, S., Bae, J., Karapınar, E.: Fixed point theorems for $\alpha$-Geraghty contraction type maps in metric spaces. Fixed Point Theory Appl. 2013, 329 (2013)

17. Karapınar, E.: A discussion on $\alpha-\psi$-Geraghty contraction type mappings. Filomat 28(4), 761-766 (2014)

18. Hilfer, R:: Applications of Fractional Calculus in Physics. Word Scientific, Singapore (2000)

19. Debnath, L.: Recent applications of fractional calculus to science and engineering. Int. J. Math. Math. Sci. 2003(54), 3413-3442 (2003)

20. Kilbas, A., Srivastava, H.M., Trujillo, J.J.: Theory and Application of Fractional Differential Equations. North Holland Mathematics Studies, vol. 204 (2006) 
21. Magin, R.L.: Fractional Calculus in Bioengineering. Begell House Publishers, Danbury (2006)

22. Caputo, M., Fabrizio, M.: A new definition of fractional derivative without singular kernel. Prog. Fract. Differ. Appl. 1, 73-85 (2015)

23. Abdeljawad, T., Baleanu, D.: Monotonicity results for fractional difference operators with discrete exponential kernels. Adv. Differ. Equ. 2017, 78 (2017)

24. Abdeljawad, T., Baleanu, D.: On fractional derivatives with exponential kernel and their discrete versions. Rep. Math. Phys. 80(1), 11-27 (2017)

25. Atangana, A., Baleanu, D.: New fractional derivative with non-local and non-singular kernel. Therm. Sci. 20, 757-763 (2016)

26. Alsaedi, A., Baleanu, D., Etemad, S., Rezapour, S.: On coupled systems of time-fractional differential problems by using a new fractional derivative. J. Funct. Spaces 2016, Article ID 4626940 (2016)

27. Agarwal, R., Baleanu, D., Hedayati, V., Rezapour, S.: Two fractional derivative inclusion problems via integral boundary condition. Appl. Math. Comput. 257, 205-212 (2015)

28. Baleanu, D., Rezapour, S., Mohammadi, H.: Some existence results on nonlinear fractional differential equations. Philos. Trans. R. Soc. 371, 20120144 (2013)

29. Baleanu, D., Mohammadi, H., Rezapour, S.: The existence of solutions for a nonlinear mixed problem of singular fractional differential equations. Adv. Differ. Equ. 2013, 359 (2013)

30. Abdeljawad, T.: Meir-Keeler $\alpha$-contractive fixed and common fixed point theorems. Fixed Point Theory Appl. 2013, 19 (2013)

31. Patel, D.K., Abdeljawad, T., Gopal, D.: Common fixed points of generalized Meir-Keeler $\alpha$-contractions. Fixed Point Theory Appl. 2013, 260 (2013)

32. Karapınar, E., Kumam, P., Salimi, P.: On $\alpha$ - $\psi$-Meir-Keeler contractive mappings. Fixed Point Theory Appl. 2013, 94 (2013)

33. Karapınar, E., Samet, B.: Generalized $\alpha-\psi$-contractive type mappings and related fixed point theorems with applications. Abstr. Appl. Anal. 2012, Article ID 793486 (2012)

34. Aydi, H., Karapınar, E., Erhan, I.M., Salimi, P.: Best proximity points of generalized almost $\psi$-Geraghty contractive non-self-mappings. Fixed Point Theory Appl. 2014, 164 (2014). https://doi.org/10.1186/1687-1812-2014-32

35. Bilgili, N., Karapınar, E., Sadarangani, K.: A generalization for the best proximity point of Geraghty-contractions J. Inequal. Appl. 2013, 286 (2013)

36. Anderson, D.R., Ulness, D.J.: Newly defined conformable derivatives. Adv. Dyn. Syst. Appl. 10(2), 109-137 (2015)

37. Khalil, R., Al Horani, M., Yousef, A., Sababheh, M.: A new definition of fractional derivative. J. Comput. Appl. Math. 264, 65-70 (2014)

38. Abdeljawad, T.: On conformable fractional calculus. J. Comput. Appl. Math. 279, 57-66 (2013)

39. Buschman, R.G.: Decomposition of an integral operator by use of Mikusinski calculus. SIAM J. Math. Anal. 3, 83-85 (1972)

40. Meerschaert, M.M., Sabzikar, F., Chen, J.: Tempered fractional calculus. J. Comput. Phys. 293, 14-28 (2015)

41. Li, C., Deng, W., Zhao, L.: Well-posedness and numerical algorithm for the tempered fractional ordinary differential equations. Discrete Contin. Dyn. Syst., Ser. B 24, 1989-2015 (2015)

42. Abdeljawad, T.: Fractional operators with generalized Mittag-Leffler kernels and their differintegrals. Chaos 29 023102 (2019). https://doi.org/10.1063/1.5085726

43. Abdeljawad, T., Baleanu, D.: Integration by parts and its applications of a new nonlocal fractional derivative with Mittag-Leffler nonsingular kernel. J. Nonlinear Sci. Appl. 10(3), 1098-1107 (2017)

\section{Submit your manuscript to a SpringerOpen ${ }^{\circ}$ journal and benefit from:}

- Convenient online submission

- Rigorous peer review

- Open access: articles freely available online

- High visibility within the field

- Retaining the copyright to your article

Submit your next manuscript at $\boldsymbol{s p r i n g e r o p e n . c o m ~}$ 